\title{
Multi-channel Broadcast via Channel Hopping in Cognitive Radio Networks
}

\author{
Lin Chen, Kaigui Bian, Member, IEEE, Xiaojiang Du, Senior Member, IEEE, \\ and Xiaoming Li, Senior Member, IEEE
}

\begin{abstract}
Broadcast is a mandatory service for wireless communications, through which the signals or contents are usually propagated as broadcasts to groups of users that subscribe to the service. However, in cognitive radio (CR) networks where secondary users opportunistically access the licensed spectrum bands, the broadcast delivery via a single broadcast channel cannot be reliably guaranteed when the broadcast channel is reclaimed by a primary (or licensed) user that has a higher priority of accessing the spectrum, or when a secondary user moves into a region where primary user transmissions are active. As a result, broadcast failures occur between the base station (BS) and the secondary user. To address this problem, the BS has to broadcast on more than one channels to avoid colliding with primary users, and ensure that all users can correctly receive the broadcasted content. In this paper, we propose a multichannel broadcast protocol, $M$ c-Broadcast, that enables a network base station to broadcast over multiple channels via a channel hopping process such that the broadcasts can be successfully delivered to secondary users. The channel hopping sequence for Mc-Broadcast is generated using a mathematical construct called Langford pairing, which allows the base station to significantly reduce the broadcast latency and given a customized number of broadcast channels. Our analytical and simulation results show that the proposed method reduces the broadcast latency and it is robust to the broadcast failure caused by primary user transmissions under various network conditions.
\end{abstract}

\section{INTRODUCTION}

Broadcast in wireless networks is typically offered as a push-type service for distributing important control information from one source to other users in the same network, e.g., from the base station to a group of users that share radio resources. Moreover, broadcast enables the low-cost delivery of large volumes of popular content (e.g., multimedia content) to users in a wireless network. There are many existing solutions to broadcast in the market, including mobile TV broadcasting (DVB-H) [12], audio casting, massive software updates, content delivery over WiMax [10], and broadcast (or multicast) service offered by $3 \mathrm{GPP}$ in LTE cellular networks [7].

A single channel, referred to as a broadcast channel, is usually used by a base station to distribute the same content to a group of users that subscribe to the same service [5].

Lin Chen is with the Department of Electrical Engineering, Yale University, New Haven, CT, USA (email: lin.chen@yale.edu). Kaigui Bian is with the Institute of Network Computing and Information Systems, School of EECS, Peking University, Beijing, China (email: bkg@pku.edu.cn). Xiaojiang Du is with the Department of Computer and Information Sciences, Temple University, Philadelphia, PA, USA (email: dux@temple.edu). Xiaoming Li is with State Key Laboratory of Software Development Environment at Beihang University, and School of EECS at Peking University, Beijing, China (email: 1xm@pku.edu.cn).
Meanwhile, a base station may employ multiple broadcast channels for delivering different contents to groups of users that subscribe to different services. A user or a content subscriber is able to successfully receive the broadcasted content when (1) the broadcast channel is available and (2) the user is located within the transmission range of the base station.

In cognitive radio (CR) networks with secondary users, the broadcast failure problem can occur due to the temporal and spatial variations in channel availability. Specifically, the primary users (PU, or licensed users) may reclaim the spectrum band where broadcast channels reside and the unlicensed users have to vacate this channel according to the requirement for protection of licensed users in cognitive radio networks. On the other hand, a secondary user is likely to move from one region where no licensed users exist into a region where licensed users are present. In either case, the broadcast channel becomes unavailable thereby leading to unsuccessful deliveries of broadcasts.

A vast majority of existing work has focused on tackling this problem in multi-hop or ad-hoc CR networks. Instead of relying on a single broadcast channel, the control information is transmitted over a pre-selected set of broadcast channels, which can be derived based on the neighbor graphs [11]. To determine the minimum broadcast schedule length for a CR network, two heuristics are presented and they can produce schedules that have either optimal or near-optimal lengths [2]. In [3], a mixed broadcast scheduling algorithm is proposed under the Unit Disk Graph (UDG) model, which combines the uni-cast and broadcast collaboratively in order to obtain a small broadcast latency. To broadcast over multiple channels, the channel hopping technique is used by cognitive radios without requiring the knowledge of global network topology or the requirement of time synchronization information [17][19].

We focus on the broadcast failure problem in an infrastructure-based (or cellular) CR network. To guarantee the successful broadcast, a base station has to employ a multi-channel broadcast protocol-i.e., it delivers contents over multiple broadcast channels using broadcast radios, so as to reduce the chance of colliding with primary users' transmissions in the spatial or temporal domain.

In an infrastructure-based CR network, the base station can be equipped with multiple radio interfaces. The rapidly diminishing prices of the radios have made it feasible to equip a wireless node with multiple radios. Providing the base station with one or more multi-channel radios offers a promising avenue for significantly reducing the latency before successful 
broadcast delivery and enhancing the network capacity by simultaneously exploiting multiple orthogonal channels through different radio interfaces and mitigating interferences through proper channel assignment. However, there is a limit (an optimal value) for the number of radios. We will show later that the optimal number of radios is twice the channel number, via both analytical and simulation results.

There are two design challenges for devising such a multichannel broadcast protocol: (1) how many broadcast channels are needed to guarantee the successful broadcast delivery? (2) what is the minimum broadcast latency given a number of broadcast channels?

In this paper, we present a multi-channel broadcast protocol, called Mc-Broadcast, for delivering contents to secondary users in an infrastructure-based CR network. Every broadcast radio at a base station selectively transmit over a number of channels via a channel hopping process. The channel hopping sequence is generated using a mathematical construct called Langford pairing, which meets the two aforementioned design challenges - the base station is free to customize the number of broadcast channels; meanwhile, the induced broadcast latency can be significantly reduced given a number of broadcast channels. Our analytical and simulation results show that McBroadcast incurs a small broadcast latency, and it guarantees a high successful delivery ratio under various network conditions.

The rest of this paper is organized as follows: we provide background knowledge in Section III. In Section IV, we present the problem formulation in the design of McBroadcast. We present the two broadcast protocols in Section V. In Section VI, we evaluate the performance of McBroadcast using simulation results. We conclude the paper in Section VII.

\section{RELATED WORK}

The problem for devising multi-channel broadcast protocols has not yet been largely studied in the context of an infrastructure-based CR network. To the best of our knowledge, only a limited number of papers have so far tackled the broadcast algorithm design problem in infrastructure-based CR networks. Nevertheless, the solutions are constructed on the basis of assumptions such as the accessibility to and availability of all possible common channels (some protocols only support ensured delivery on a subset of common channels), or the existence of a global (network-wide) or local common broadcast/control channel. In [21], Yang et al. proposed a deterministic rendezvous scheme that leverages the deterministic rendezvous sequence (DRSEQ). In [15], Lin et al. proposed a jump-stay based channel hopping protocol for guaranteed broadcast delivery. A network that uses DRSEQ or the jump-stay based algorithm can ensure successful delivery despite any amount of clock drift between the BS and user nodes. However, neither of them can guarantee broadcast delivery on all possible channels. If primary user activity occupies a subset of common channels that both the BS and the user node can sense, a channel hopping algorithm that fails to guarantee broadcast delivery on all possible channels will not enable the BS and the user node to establish delivery links on the rest of common channels that have not yet been occupied due to primary user traffic. Arachchige et al. proposed a broadcast protocol on the basis of Integer Linear Programming (ILP) [1]. However, the proposed solutions in [1] impractically assume a common signaling channel for the whole network. The use of a single common control channel simplifies the broadcast delivery establishment process but it creates a single point of failure-it may become unavailable due to the appearance of licensed user signals and furthermore a broadcast protocol based on a single common control channel is subject to the jamming problem. In [14], Lazos et al. proposed a distributed cluster agreement algorithm called Spectrum-Opportunity Clustering (SOC) that aims to locally establish a common control channel in each cluster and thus may be regarded as an alternative. However, SOC requires apriori knowledge regarding spectrum sensing capabilities of all secondary nodes, which can be obtained only after some message exchanges happen. In [4], Cormio et al. proposed a scheme that allows altering the hopping sequence based on the PU activity in the channels so as to find a common control channel, while they become infeasible due to some inherent limitations in various and complicated network environments. In view of these drawbacks, the nodes fail to ensure successful broadcast delivery under certain conditions, which rules it out in broadcast scenarios. In [19], a distributed broadcast protocol without assuming a common control channel is proposed. However, it is devised for multi-hop cognitive radio ad hoc networks while this paper delves into the issues in infrastructure-based CR networks.

\section{TeChNicAL BACKGROUND}

In this section, we provide the background knowledge relevant to the Langford Pairing (LP) [13] and the Extended Langford Pairing (ELP).

\section{A. Langford Pairing}

Definition of LP. Given an integer $n$, Langford pairing is a sequence of length $2 n$ that consists of two 1's, two 2's, ..., and two $n$ 's and satisfies that there are exactly one number between the two 1's, exactly two numbers between the two 2 's, ..., and exactly $n$ numbers between the two $n$ 's.

Formally, A Langford pairing, $\left\{l_{i}\right\}_{0 \leq i \leq 2 n-1}$ of order $n$, also called a Langford sequence, is a permutation of the sequence of $2 n$ integers $\{1,1,2,2,3,3, \ldots, n, n\}$, and it satisfies the Langford property: if $l_{i}=l_{j}, 0 \leq i<j \leq 2 n-1$, then $j-i=l_{i}+1$.

For example, the sequence $l=\{3,1,2,1,3,2\}$ is a Langford pairing of order $n=3$. There is only one number (that is 2 ) between the two 1's, two numbers (they are 1 and 3) between the two 2's and three numbers (they are 1,2 and 1) between the two 3's. Given $i=0$ and $j=4$, we have $l_{0}=l_{4}=3$, and $j-i=3+1$; given other combinations of $i$ and $j$, the sequence $l$ also satisfies the Langford property.

With respect to the existence of Langford pairing of given order $n$, the following lemma gives a sufficient and necessary condition. 


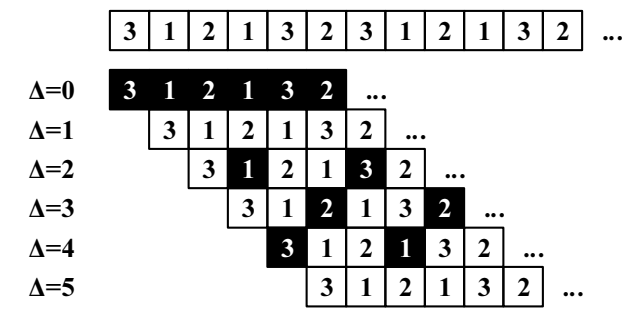

Figure 1: We put two copies of the Langford pairing $\{3,1,2,1,3,2\}$ together and shift one of them by $\Delta$ grids. This figure illustrates different situations with $\Delta$ ranging from 0 to 5 .

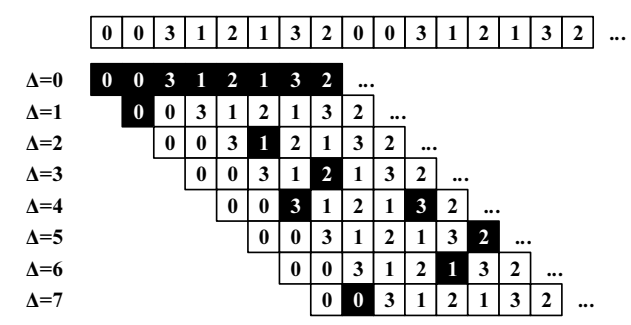

Figure 2: We put two copies of the extended Langford pairing $\{0,0,3,1,2,1,3,2\}$ together and shift one of them by $\Delta$ grids. This figure illustrates different situations with $\Delta$ ranging from 0 to 7 .

Lemma 1. A Langford pairing of order $n$ exists if and only if $n$ is congruent to 0 or 3 modulo 4.

For the proof of Lemma 1, please refer to the Theorem 1 in [16].

Drawback of LP and motivation of ELP. In Fig. 1, we put two copies of the Langford pairing $\{3,1,2,1,3,2\}$ together and shift one of them by $\Delta$ grids. Fig. 1 illustrates different situations with $\Delta$ ranging from 0 to 5 . According to the construction of Langford pairing, there are 1 number between the two 1's, when $\Delta=1+1=2$, the second copy's 2nd grid with a " 1 " inside aligns with the first copy's 4th grid with a "1" inside. Since there are 2 numbers between the two 2's, when $\Delta=2+1=3$, the second copy's 3rd grid with a "2" inside aligns with the first copy's 6th grid with a "2" inside. We hope that for all possible $\Delta$ 's, there always exist two aligned grids of the two copies with the same number inside the grids. In Fig. 1, this is false when $\Delta=1$ or $\Delta=5$. This is because there are no neighboring positions with the same number in an LP. Thus we insert two 0's at the beginning of an LP and get an extended LP $\{0,0,3,1,2,1,3,2\}$. In Fig. 2 , we can observe that for all possible $\Delta$ 's, there always exist two aligned grids in the two copies with the same number inside the two grids. In Sec. III-B, we introduce the notion of the extended LP.

\section{B. Extended Langford Pairing (ELP)}

Slightly different from LP, we require that an ELP contain two 0's (note that an LP does not contain any 0's) and that these two 0's be neighboring. Formally, we define an extended Langford pairing, $\left\{l_{i}^{\prime}\right\}_{0 \leq i \leq 2(n+1)-1}$ of order $n$, as a permutation of the sequence of $2(n+1)$ integers:

$$
\{0,0,1,1,2,2, \ldots, n, n\} .
$$

The sequence satisfies the Langford property, i.e., if $l_{i}=$ $l_{j}, 0 \leq i<j \leq 2(n+1)-1$, then $j-i=l_{i}+1$.

For example, the sequence

$$
l^{\prime}=\{0,0,3,1,2,1,3,2\},
$$

is an extended Langford pairing (ELP) of order $n=3$.

Given a Langford pairing $\left\{l_{i}\right\}_{0 \leq i \leq 2 n-1}$ of order $n$, we can easily construct an extended Langford pairing $\left\{l_{i}^{\prime}\right\}_{0 \leq i \leq 2(n+1)-1}$ of the same order by inserting two integers $l_{0}^{\prime}=\bar{l}_{1}^{\prime}=0$ at the beginning of the original LP-i.e., by letting $l_{i}^{\prime}=0$ when $i=0,1$ and $l_{i}^{\prime}=l_{i-2}$ when $1<i \leq 2 n+1$. It follows immediately from Lemma 1 that an extended Langford pairing of order $n$ exists if $n$ is congruent to 0 or 3 modulo 4.

Given an extended Langford pairing, $l^{\prime}$, of order $n$, any integer $k \in[0, n]$ appears exactly twice in the ELP. Suppose $l_{i}^{\prime}=l_{j}^{\prime}=k$ and $i<j$, we define $l_{i}^{\prime}$ and $l_{j}^{\prime}$ as the $k$-valued pair in the ELP, and $d(k)=j-i$ denotes the distance between the two integers in the $k$-valued pair. As a result, we have the following lemma.

Lemma 2. Given an extended Langford pairing, $l^{\prime}$, of order $n$, there exists exactly one $k$-valued pair, for each $k \in[0, n]$.

Inspired by the conclusion in Lemma 2, we use ELP to generate channel hopping sequences for the base station and users, which will be described in Section V.

\section{Problem Formulation}

\section{A. System Model}

1) Multi-channel broadcast: In a CR network, the BS and the users in the BS's service area are secondary users, and they are equipped with CRs operating over broadcast channels that are licensed to the primary user (PU). Due to PU's activities, a broadcast channel may become unavailable at any time. Therefore, the BS has to broadcast the content over multiple channels to ensure the successful delivery to users, and we call such a process as a multi-channel broadcast process.

Suppose there are $N$ broadcast channels, labeled as $0,1,2, \ldots, N-1$. The BS is equipped with multiple broadcast radios, labeled as $r_{1}, r_{2}, \ldots, r_{R}$, where $R$ is the total number of the BS's broadcast radios. There are $U$ users $s_{1}, s_{2}, \ldots, s_{U}$ in the service area. Every user is equipped with a single radio interface.

2) Broadcast via channel hopping $(\mathrm{CH})$ : To implement the multi-channel broadcast protocol, a BS's broadcast radio or a user's radio can hop across multiple broadcast channels to deliver or to receive the broadcast content. Thus, we use the channel hopping $(\mathrm{CH})$ sequence to define the order with which a BS's broadcast radio (or a user's radio) visits the set of broadcast channels.

We consider a time-slotted communication system, where a global system clock exists. The local clock of each node may be synchronized to the global clock or may differ with the global clock by a certain amount of clock drift. A radio is 
assumed to be capable of hopping between different channels according to a channel hopping sequence and its local clock. A packet can be exchanged between two radios if they hop onto the same channel in the same timeslot.

Then, we represent a $\mathrm{CH}$ sequence $u$ of period $T$ as a sequence of channel indices:

$$
u=\left\{u_{0}, u_{1}, u_{2}, \ldots, u_{i}, \ldots, u_{T-1}\right\},
$$

where $u_{i} \in[0, N-1]$ represents the channel index of the $i$ th timeslot of $\mathrm{CH}$ sequence $u$. If $u_{i}=u_{j}, \forall i, j \in[0, T-1]$, the radio using $u$ as its $\mathrm{CH}$ sequence stays on the same channel and does not hop.

Given two $\mathrm{CH}$ sequences of period $T, u$ and $v$, if there exists $i \in[0, T-1]$ such that $u_{i}=v_{i}=h$, where $h \in[0, N-1]$, we say that a broadcast delivery occurs between $u$ and $v$ in the $i$ th timeslot on broadcast channel $h$. The $i$ th timeslot is called a delivery slot and channel $h$ is called a delivery channel between $u$ and $v$.

Given $N$ channels, let $\mathcal{C}(u, v)$ denote the set of delivery channels between two $\mathrm{CH}$ sequences $u$ and $v$. The cardinality of $\mathcal{C}(u, v)$ is called the number of broadcast delivery channels, denoted by $|\mathcal{C}(u, v)|$, and $|\mathcal{C}(u, v)| \in[0, N]$. The number of broadcast delivery channels measures the number of channels in which successful broadcast delivery occurs, i.e. the diversity of broadcast delivery channels.

Let $\mathcal{T}(u, v)$ denote the set of delivery slots between two $\mathrm{CH}$ sequences $u$ and $v$, and $|\mathcal{T}(u, v)| \in[0, T]$. The cardinality of $\mathcal{T}(u, v)$ reflects the number of timeslots in which successful broadcast delivery occurs within a period.

3) Broadcast by multiple radios: To reduce the broadcast latency, the BS is allowed to use a set of broadcast radios, denoted by $\mathcal{B}=\left\{r_{1}, r_{2}, r_{3}, \ldots, r_{R}\right\}$. Since the BS has multiple radios, the broadcast delivery occurs between the BS and a user $s$ if the broadcast delivery occurs between one of the BS's broadcast radios and the user $s$ 's radio - i.e. the broadcast delivery occurs between a radio set $\mathcal{B}$ and a user $s$ if there exists a radio $r_{i} \in \mathcal{B}$ such that a broadcast delivery occurs between the $\mathrm{CH}$ sequences of radios $r_{i}$ and $s$. To simplify the notation, we simply use $r_{i}$ to denote the $\mathrm{CH}$ sequence of the BS's broadcast radio $r_{i} \in \mathcal{B}$ and use $s$ to denote the $\mathrm{CH}$ sequence of user $s$ 's radio.

The set of broadcast delivery channels between the BS with its set of broadcast radios $\mathcal{B}$ and a user $s$ is the union of the sets of broadcast delivery channels between each broadcast radio of the BS and the user $s$ 's radio, i.e. let $\mathcal{C}(\mathcal{B}, s)=\bigcup_{r \in \mathcal{B}} \mathcal{C}(r, s)$ denote the set of broadcast delivery channels between the BS with its set of broadcast radios $\mathcal{B}$ and the user $s$ 's radio, and the cardinality of $\mathcal{C}(\mathcal{B}, s)$ is called the number of delivery channels, denoted by $|\mathcal{C}(\mathcal{B}, s)|$, and $|\mathcal{C}(\mathcal{B}, s)| \in[0, N]$.

Similarly, the set of delivery slots between the BS with its set of broadcast radios $\mathcal{B}$ and a user $s$ is the union of the sets of delivery slots between each broadcast radio of the BS and the user $s$ 's radio, i.e. let $\mathcal{T}(\mathcal{B}, s) \triangleq \bigcup_{r \in \mathcal{B}} \mathcal{T}(r, s)$ denote the set of delivery slots between the BS with its set of broadcast radios $\mathcal{B}$ and the user $s$ 's radio, and $|\mathcal{T}(\mathcal{B}, s)| \in[0, T]$.

4) An asynchronous multi-channel broadcast system: Given a $\mathrm{CH}$ sequence $u$, we use $\operatorname{rotate}(u, k)$ to denote a cyclic rotation of $\mathrm{CH}$ sequence $u$ by $k$ timeslots, i.e.,

$$
\operatorname{rotate}(u, k)=\left\{v_{0}, \ldots, v_{j}, \ldots v_{T-1}\right\},
$$

where $v_{j}=u_{(j+k)} \bmod T, j \in[0, T-1]$. For example, given $u=\{0,1,2\}$ and $T=3$, rotate $(u, 2)=\operatorname{rotate}(u,-1)=$ $\{2,0,1\}$.

We define an asynchronous multi-channel broadcast $(A M B)$ system $\mathcal{M}$ with $\mathrm{CH}$ period $T$ as an ordered pair $(\mathcal{B}, \mathcal{U})$ :

- $\mathcal{B}$ is the set of $\mathrm{CH}$ sequences of period $T$ used by broadcast radios of the BS. Suppose $\mathcal{B}=\left\{r_{1}, r_{2}, r_{3}, \ldots, r_{R}\right\}$, where $R$ is the number of the BS's broadcast radios, and the BS's broadcast radio $r_{i}$ uses the $\mathrm{CH}$ sequence $r_{i}$ in $\mathcal{B}$.

- $\mathcal{U}$ is the set of $\mathrm{CH}$ sequences of period $T$ used by the users. Suppose $\mathcal{U}=\left\{s_{1}, s_{2}, s_{3}, \ldots, s_{U}\right\}$, where $U$ is the number of users, and the user $s_{j}$ 's radio uses the $\mathrm{CH}$ sequence $s_{j}$ in $\mathcal{U}$.

Recall that we simply use $r_{i}$ and $s_{j}$ to denote the $\mathrm{CH}$ sequences of the BS's broadcast radio $r_{i} \in \mathcal{B}$ and user $s_{j}$ 's radio, respectively.

An AMB system must satisfy the rotation closure property: $\forall k, l \in[0, T-1], \forall s \in \mathcal{U}$, there exists $r \in \mathcal{B}$ such that $\mid \mathcal{C}($ rotate $(s, k)$, rotate $(r, l)) \mid \geq 1$. And thus we map the design problem of $\mathrm{CH}$ sequences of the BS's broadcast radios and users' radios to the design problem of an $\mathrm{AMB}$ system with rotation closure property. And the rotation closure property implies that for all possible clock drifts between the BS and users, every user can have successful broadcast delivery with the BS, i.e. with one of the BS's broadcast radios.

In other words, in an AMB system, the BS, with each broadcast radio using $\mathrm{CH}$ sequences in $\mathcal{B}$, can deliver broadcast messages to all users using $\mathrm{CH}$ sequences in $\mathcal{U}$ via a channel hopping process for all possible clock drifts.

\section{B. Performance Metrics}

Given an $\mathrm{AMB}$ system $\mathcal{M}=(\mathcal{B}, \mathcal{U})$, we introduce the following metrics for evaluating its performance.

- Delivery channel diversity. The delivery channel diversity for an AMB system measures the lower bound of the number of delivery channels between the BS and an arbitrarily given user for all possible clock drifts. The delivery channel diversity, denoted by $D I V(\mathcal{M})$, is the minimum number of delivery channels $\mid \mathcal{C}(\mathcal{B}$, rotate $(s, k)) \mid$ for every $s \in \mathcal{U}$ and every $k \in \mathbb{Z}$, i.e.,

$$
\begin{aligned}
D I V(\mathcal{M}) & =\min _{s \in \mathcal{U}, k \in \mathbb{Z}} \mid \mathcal{C}(\mathcal{B}, \text { rotate }(s, k)) \mid \\
& =\min _{s \in \mathcal{U}, k \in \mathbb{Z}} \mid \bigcup_{r \in \mathcal{B}} \mathcal{C}(r, \text { rotate }(s, k)) \mid .
\end{aligned}
$$

- Broadcast latency. To quantify the broadcast latency, we define the maximum broadcast latency for a given AMB system as the upper bound of the latency before the first successful broadcast delivery between the BS and an arbitrary user on at least one channel for all possible clock drifts, which can be computed by

$$
\max _{s \in \mathcal{U}, k \in \mathbb{Z}}[\min \mathcal{T}(\mathcal{B}, \operatorname{rotate}(s, k))] .
$$


- Delivery ratio. To measure the proportion of delivery slots in a period, we define the delivery ratio for a $\mathrm{CH}$ sequence pair. The delivery ratio for a $\mathrm{CH}$ sequence pair $r$ and $s$, denoted by $\rho(r, s)$, is

$$
\min _{k, l \in \mathbb{Z}}(\mid \mathcal{T}(\text { rotate }(r, k), \text { rotate }(s, l)) \mid / T) .
$$

And then we introduce the delivery ratio for an AMB system $\mathcal{M}=(\mathcal{B}, \mathcal{U})$, which measures the minimum proportion of delivery slots in all timeslots. To be precise, the delivery ratio is

$$
\rho(\mathcal{M}) \triangleq \min _{s \in \mathcal{U}, k, l \in \mathbb{Z}} \frac{\sum_{r \in \mathcal{B}} \mid \mathcal{T}(\text { rotate }(r, k), \text { rotate }(s, l)) \mid}{|\mathcal{B}| T} .
$$

\section{The Optimal AMB System}

To reduce the chance of broadcast failure due to the presence of primary user signals, a BS needs to maximize the number of distinct delivery channels (i.e. achieve full delivery channel diversity). We would ideally want an AMB system to guarantee $N$ distinct delivery channels between a BS's radio and any users when $N$ channels are available.

Our objective is to devise an optimal AMB system as a system that has the maximum delivery channel diversity, minimum broadcast latency, and the maximum delivery ratio during a period.

\section{Extended LANGFord PAIRING BASEd BRoAdCAST PROTOCOLS}

In this section, we construct an $\mathrm{AMB}$ system $\mathcal{M}=(\mathcal{B}, \mathcal{U})$ based on the Extended Langford Pairing (ELP). To illustrate our design of the ELP-based AMB system, we first investigate a simple scenario in which the BS has a single radio, i.e., $|\mathcal{B}|=1$, and there is only a single user, i.e., $|\mathcal{U}|=1$. Then we address the general scenario where $|\mathcal{B}|$ and $|\mathcal{U}|$ are generally greater than 1 .

\section{A. Fundamentals of ELP-based CH Sequences}

In this subsection, we mainly discuss the ELP-based channel hopping protocol for AMB systems with a single radio pair, i.e., $\mathcal{M}=(\{r\},\{s\})$, where $r$ is the only BS radio and $s$ is the only user.

1) $\mathrm{CH}$ sequence generation: We focus on the simple scenario where $|\mathcal{B}|=|\mathcal{U}|=1$, i.e., $\mathcal{B}=\{r\}$ and $\mathcal{U}=\{s\}$. By investigating the simple scenario, we establish mathematical properties of ELP that are pivotal for the design of a general AMB system that is to be discussed later.

To begin with, we explain why ELP rather than the orginal LP is leveraged. We consider the original Langford Pairing (LP). If $N$ is congruent to 0 or 3 modulo 4 , by Lemma 1 , there exists an LP $\left\{l_{i}\right\}_{0 \leq i \leq 2 N-1}$ of order $N$. Suppose both $r$ and $s$ use the CH sequence $\left\{l_{i}-1\right\}_{0 \leq i \leq 2 N-1}$ of period $2 N$. If $s$ is one timeslot ahead, the broadcast delivery cannot occur between $r$ and $s$. For example, suppose the channel number $N=3 \equiv 3(\bmod 4)$ and $\{3,1,2,1,3,2\}$ is an LP of order 3 . Both $r$ and $s$ use the $\mathrm{CH}$ sequence $\{2,0,1,0,2,1\}$ of period 6. If $s$ is one timeslot ahead, the broadcast delivery cannot occur between $r$ and $s$, i.e., $\mid \mathcal{C}(r$, rotate $(s, 1)) \mid=0$.
However, we can construct an AMB system by using ELP. If the channel number $N$ is congruent to 0 or 1 modulo 4 , then $N-1$ is congruent to 0 or 3 , and by Lemma 1 , there exists an ELP $\left\{l_{i}^{\prime}\right\}_{0 \leq i \leq 2 N-1}$ of order $N-1$. For example, when $N=4$, the ELP-based $\mathrm{CH}$ sequence is

$$
u=\{0,0,3,1,2,1,3,2\} .
$$

When $N \not \equiv 0,1(\bmod 4)$, we can easily use the downsizing scheme or the padding scheme to transform it into an AMB system design problem with the channel number $N^{\prime}$ congruent to 0 or 1 modulo 4 .

Downsizing scheme. Suppose the channel number $N \not \equiv 0,1$ $(\bmod 4)$, let $N^{\prime}$ be $\max \left\{N^{\prime} \leq N: N^{\prime} \equiv 0,1(\bmod 4)\right\}$. It is easy to see that $\left|N-N^{\prime}\right| \leq 2$. The donwsizing scheme will limit the set of broadcast channels to a $N^{\prime}$-element subset of the original broadcast channel set, e.g., we use $\left\{0,1,2, \ldots, N^{\prime}-1\right\}$ as the new broadcast channel set.

Padding scheme. Suppose the channel number $N \not \equiv 0,1$ $(\bmod 4)$, let $N^{\prime}$ be $\min \left\{N^{\prime} \geq N: N^{\prime} \equiv 0,1(\bmod 4)\right\}$. It is easy to see that $\left|N-N^{\prime}\right| \leq 2$. In contrast with the downsizing scheme, the padding scheme introduces $N^{\prime}-N$ more channels but maps them to the original broadcast channels in $\{0,1,2, \ldots, N-1\}$. For example, suppose $N=7$ and we have $N^{\prime}=8$. Now we introduce one more channel, i.e. channel 7 , and adjoin channel 7 to the original broadcast channel set $\{0,1,2, \ldots, 6\}$ but channel 7 is an alias of channel 0 - i.e. it is mapped to channel 0 .

For instance, if $N \equiv 2(\bmod 4)$, we can use the downsizing scheme and $\left|N-N^{\prime}\right|=1$; if $N \equiv 3(\bmod 4)$, we can use the padding scheme and $\left|N-N^{\prime}\right|=1$. And this will only lead to a very mild degradation of performance since $\left|N-N^{\prime}\right|=1$.

With the aid of the downsizing scheme and the padding scheme, we can focus on the AMB design problem with the channel number $N^{\prime}$ congruent to 0 or 1 modulo 4 .

2) Properties of ELP CH sequences: In an AMB system with a single radio pair (the BS has only one broadcast radio $r$ and there is only one user $s$ ), suppose both $r$ and $s$ use the ELP $\left\{l_{i}^{\prime}\right\}_{0 \leq i \leq 2 N^{\prime}-1}$ of period $2 N^{\prime}$ as their $\mathrm{CH}$ sequences, where $N^{\prime} \equiv 0,1(\bmod 4)$. Theorem 3 shows that the broadcast delivery between $r$ and $s$ can always occur for all possible clock drifts and that the set of broadcast delivery channels is determined by the clock drift (in the statement of Theorem 3, $k-l$ is the clock drift). Theorem 3 is the basis of achieving delivery channel diversity that will be addressed later in that we can manipulate the set of broadcast delivery channels by deliberately manipulating the clock drift.

Theorem 3. $u=\left\{l_{i}^{\prime}\right\}_{0 \leq i \leq 2 N^{\prime}-1}$ is an ELP of order $N^{\prime}-1$.

1) If $k-l \equiv 0\left(\bmod 2 N^{\prime}\right)$, then $\operatorname{rotate}(u, k)=$ rotate $(u, l)$ and $\mathcal{C}($ rotate $(u, k)$, rotate $(u, l))=$ $\left\{0,1,2, \ldots, N^{\prime}-1\right\}$,

2) If $k-l \equiv g \not \equiv 0\left(\bmod 2 N^{\prime}\right)$, where $|g| \leq N^{\prime}$, then $\mathcal{C}(\operatorname{rotate}(u, k)$, rotate $(u, l))=\{|g|-1\}$.

The following shows an immediate corollary that for all possible clock drifts, the two radios using the same ELP will always have successful broadcast delivery. 
Corollary 4. $u=\left\{l_{i}^{\prime}\right\}_{0 \leq i \leq 2 N^{\prime}-1}$ is an ELP of order $N^{\prime}-1 . \forall k, l \in \mathbb{Z}, \mid \mathcal{C}($ rotate $(u, k)$, rotate $(u, l)) \mid \geq 1$, i.e., rotate $(u, k)$ and rotate $(u, l)$ can always have at least one delivery slot.

Theorem 5 discusses the relation between the clock drift, i.e. $k-l$, and the set of delivery slots. It shows that the cardinality of the set of delivery slots can only be 1,2 and $2 N^{\prime}$. Note that the cardinality of the set of delivery slots is always greater than 1, i.e., the broadcast delivery can always occur between $r$ and $s$ if they use the same ELP.

Theorem 5. $u=\left\{l_{i}^{\prime}\right\}_{0 \leq i \leq 2 N^{\prime}-1}$ is an ELP of order $N^{\prime}-1$.

1) If $k-l \equiv 0\left(\bmod 2 N^{\prime}\right)$, then $\mathcal{T}(\operatorname{rotate}(u, k), \operatorname{rotate}(u, l))=\left\{0,1,2,3, \ldots, 2 N^{\prime}-\right.$ $1\}$.

2) If $k-l \equiv g \not \equiv 0\left(\bmod 2 N^{\prime}\right)$, where $|g| \leq N^{\prime}$, then

a) If $|g|<N^{\prime}$, then $\mid \mathcal{T}$ (rotate $(u, k)$, rotate $\left.(u, l)\right) \mid=$ 1.

b) If $|g|=N^{\prime}$, then $\mid \mathcal{T}(\operatorname{rotate}(u, k)$, rotate $(u, l)) \mid=$ 2 .

\section{B. A Simple Broadcast (S-Broadcast) Scheme for a Single $\mathrm{CH}$ Sequence Pair}

In this subsection, we propose a simple broadcast scheme, called $S$-Broadcast, for the simple scenario of the AMB system $(\mathcal{M}=(\{r\},\{s\}))$ design problem.

Motivation. By Theorem 3, if the broadcast radio $r$ and the user radio $s$ both use the same ELP $u=\left\{l_{i}^{\prime}\right\}_{0 \leq i \leq 2 N^{\prime}-1}$, the successful broadcast delivery between them is guaranteed, however, the delivery channel diversity is not ensured. If the broadcast radio uses cyclic rotated copies of $u$ and the user radio uses periodically extended $u$, the delivery channel diversity will be increased.

To be precise, the $\mathrm{CH}$ sequences for the broadcast and user radios can be generated as follows:

1) The broadcast radio generates its $\mathrm{CH}$ sequence

$$
r=\prod_{f=1}^{F} \operatorname{rotate}\left(u, o_{f}\right),
$$

where $\left\{o_{f}\right\}_{1 \leq f \leq F}$ is a sequence of integers that are used to deliberately manipulate the clock drift and $\prod_{f=1}^{F} \theta_{f}$ denotes the concatenation of strings $\theta_{1}, \theta_{2}, \theta_{3}, \ldots, \theta_{F}$, i.e., $\prod_{f=1}^{F} \theta_{f}=\theta_{1}\left\|\theta_{2}\right\| \theta_{3}\|\cdots\| \theta_{F}$;

2) The user radio generates its $\mathrm{CH}$ sequence as $s=$ $\prod_{f=1}^{F} u$, which is the periodic extension of $u$.

We define the delivery channel determination function such as

$$
\delta: \mathbb{Z} \rightarrow \mathbb{Z}
$$

For $k \in \mathbb{Z}, k \equiv g\left(\bmod 2 N^{\prime}\right)$ where $|g| \leq N^{\prime}$, then $\delta(k)=$ $|g|-1$. For example, when $N^{\prime}=4, \delta(1)=0, \delta(2)=1$, $\delta(3)=2, \delta(4)=3, \delta(5)=2, \delta(6)=1, \delta(7)=0$, and in particular $\delta(0)=-1$.

Theorem 6 shows the estimation of delivery channel diversity of the AMB system $\mathcal{M}=(\{r\},\{s\})$.
Theorem 6. $u=\left\{l_{i}^{\prime}\right\}_{0 \leq i \leq 2 N^{\prime}-1}$ is an ELP of order $N^{\prime}-1$. $r=\prod_{f=1}^{F}$ rotate $\left(u, o_{f}\right), s=\prod_{f=1}^{F} u$, where $\left\{o_{f}\right\}_{1 \leq f \leq F}$ is a sequence of integers.

1) If $\forall k \in \mathbb{Z}, \exists 1 \leq f \leq F$, s.t. of $-k \equiv 0\left(\bmod 2 N^{\prime}\right)$, then the delivery channel diversity for the $A M B$ system $\mathcal{M}=(\{r\},\{s\})$ is $N^{\prime}$.

2) If $\exists k_{0} \in \mathbb{Z}$, s.t. $\forall 1 \leq f \leq F, o_{f}-k_{0} \not \equiv 0\left(\bmod 2 N^{\prime}\right)$, then the delivery channel diversity $D I V(\mathcal{M})$ for the $A M B$ system $\mathcal{M}=(\{r\},\{s\})$ satisfies $\min _{k \in \mathbb{Z}} \mid\left\{\delta\left(o_{f}-\right.\right.$ $k)\}_{1 \leq f \leq F}|\leq D I V(\mathcal{M}) \leq|\left\{\delta\left(o_{f}-k_{0}\right)\right\}_{1 \leq f \leq F} \mid \leq F$.

S-Broadcast. Our proposed broadcast protocol for the simple scenario of the AMB system $(\mathcal{M}=(\{r\},\{s\}))$ design problem, S-Broadcast, is an asynchronous $\mathrm{CH}$-based channel broadcast protocol that achieves the broadcast latency at most $2 N^{\prime}-1$, the delivery ratio $\rho=\frac{1}{N^{\prime}}$ and full diversity. According to the design of S-Broadcast,

$$
\text { - } r=\prod_{f=1}^{2 N^{\prime}} \operatorname{rotate}(u, f-1) \text {. }
$$

Two examples illustrating the $\mathrm{CH}$ sequences of S-Broadcast when $N^{\prime}=4$ are shown in Figure 3.

Theorem 7 shows the performance metrics of S-Broadcast. We will prove that an AMB system that implements SBroadcast has bounded broadcast latency and can achieve full delivery channel diversity. In addition, we calculate its delivery ratio.

Theorem 7. $\mathcal{M}=(\{r\},\{s\})$ is an $A M B$ system that implements $S$-Broadcast. Then

1) The broadcast latency is at most $2 N^{\prime}-1$.

2) The delivery ratio $\rho=\frac{4 N^{\prime}}{4 N^{\prime 2}}=\frac{1}{N^{\prime}}$.

3) The delivery channel diversity is $N^{\prime}$.

\section{A Multi-channel Broadcast (Mc-Broadcast) Scheme for Multiple CH Sequence Pairs}

In the general $\mathrm{AMB}$ system design problem, $|\mathcal{B}|$ and $|\mathcal{U}|$ are generally greater than 1 , i.e., $\mathcal{B}=\left\{r_{1}, r_{2}, r_{3}, \ldots, r_{R}\right\}$, $\mathcal{U}=\left\{s_{1}, s_{2}, s_{3}, \ldots, s_{U}\right\}, R, U \geq 1$. In this subsection, we first design two ELP-based CH protocols, A-Broadcast and $L$-Broadcast, for the case $R \geq 2 N^{\prime}$ and the case $R<2 N^{\prime}$, respectively. In Theorems 9 and 10, we theoretically evaluate their performance.

Then, we propose a multi-channel broadcast protocol, called Mc-Broadcast, that combines both of the two above ELP-based $\mathrm{CH}$ protocols-i.e., it adopts A-Broadcast if $R \geq 2 N^{\prime}$ and it adopts L-Broadcast if $R<2 N^{\prime}$.

Suppose $u=\left\{l_{i}^{\prime}\right\}_{0 \leq i \leq 2 N^{\prime}-1}$ is an ELP of order $N^{\prime}-1$. Theorem 3 shows that broadcast delivery can occur between any two cyclic rotation copies of $u$, say, between rotate $(u, k)$ and $\operatorname{rotate}(u, l)$-i.e., if $\forall 1 \leq i \leq R, r_{i}=\operatorname{rotate}\left(u, o_{i}\right)$, and $\forall 1 \leq j \leq U, s_{j}=u, \mathcal{M}=(\mathcal{B}, \mathcal{U})$ is an AMB system. However, full delivery channel diversity $\left(D I V(\mathcal{M})=N^{\prime}\right)$ is not necessarily guaranteed and we expect to reduce the broadcast latency. To take the advantage of multiple broadcast radios of the $\mathrm{BS}$, it would be beneficial to select $o_{i}$ 's properly so as to achieve full delivery channel diversity, reduce the broadcast latency down to zero and guarantee successful broadcast delivery in every timeslot. 


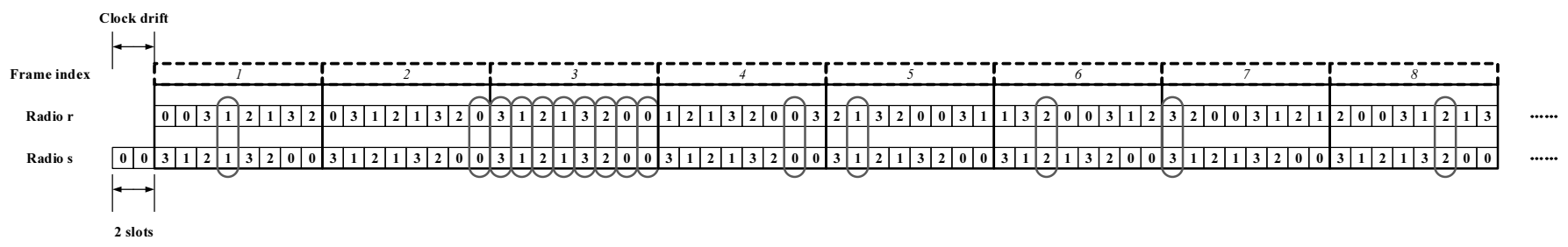

(a)

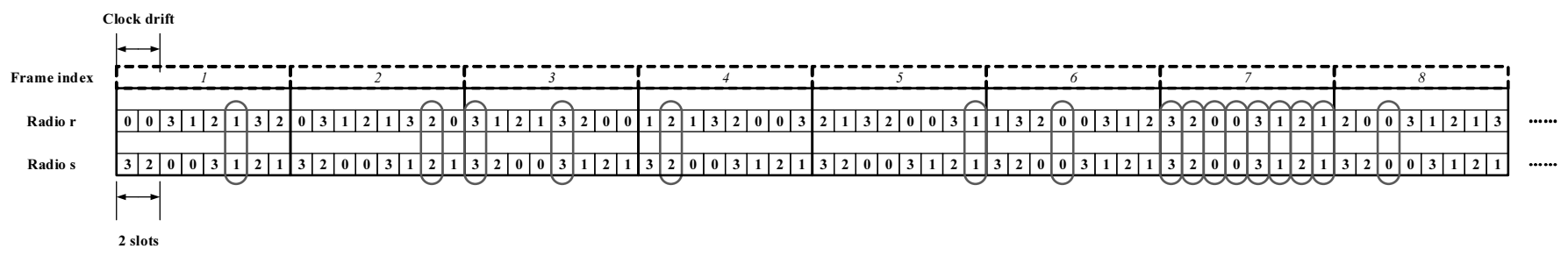

(b)

Figure 3: Example illustrating the $\mathrm{CH}$ sequences of S-Broadcast when $N^{\prime}=4$. Radio $r$ is the BS's broadcast radio and there is only one user, i.e. radio $s$. We can observe that the $\mathrm{CH}$ sequences of the two radios can achieve full diversity within a period of $4 N^{\prime 2}=64$ timeslots, given a clock drift of two slots either forwards or backwards. The delivery latency in Fig. 3a and 3b is 3 and 5 timeslots, respectively, both less than $2 N^{\prime}-1=7$. And the delivery ratios in these two figures are both $\frac{1}{N^{\prime}}=\frac{1}{4}$.

We define the balance sequences

$$
\psi_{i}^{n, k}(0 \leq i<\operatorname{lcm}(n, k) / k),
$$

where $\psi_{i}^{n, k}$ is a sequence of $k$ elements and $\operatorname{lcm}(n, k)$ is the least common multiple of $n$ and $k$. We denote the $j$-th element in $\psi_{i}^{n, k}$ by $\psi_{i, j}^{n, k}$, where $0 \leq j<k$. And let

$$
\psi_{i, j}^{n, k} \triangleq(i k+j) \bmod n \in[0, n-1] .
$$

It is easy to see that $\{i k+j \mid 0 \leq i<\operatorname{lcm}(n, k) / k, 0 \leq j<$ $k\}=\{0,1,2,3, \ldots \operatorname{lcm}(n, k)-1\}$. Hence $\forall n_{0} \in[0, n-1]$, there exist exactly $\frac{\operatorname{lcm}(n, k)}{n}(i, j)$-pairs such that $\psi_{i, j}^{n, k}=(i k+$ $j) \bmod n=n_{0}$. As a result, we have the following lemma.

Lemma 8. $\forall n_{0} \in[0, n-1]$, there exist exactly $\frac{\operatorname{lcm}(n, k)}{n}(i, j)$ pairs such that $\psi_{i, j}^{n, k}=(i k+j) \bmod n=n_{0}$.

1) A-Broadcast when $R \geq 2 N^{\prime}$ : If the BS has a large number of broadcast radios, i.e., $R \geq 2 N^{\prime}$, the AMB system can be designed to have guaranteed successful broadcast delivery in every timeslot and full delivery channel diversity. Suppose $R=2 q N^{\prime}+w$, where $q=\left\lfloor\frac{R}{2 N^{\prime}}\right\rfloor \geq 1$ and $0 \leq w<2 N^{\prime}$. We design a protocol, called A-Broadcast, as follows:

- $\forall 1 \leq i \leq 2 q N^{\prime}, r_{i}=\operatorname{rotate}\left(u,(i-1) \bmod 2 N^{\prime}\right)$.

- $\forall 1 \leq i \leq w$, from timeslot $\left\lfloor\frac{t_{B}}{2 N^{\prime}}\right\rfloor$ to timeslot $\left\lfloor\frac{t_{B}}{2 N^{\prime}}\right\rfloor+$ $\left(2 N^{\prime}-1\right)$, radio $r_{2 q N^{\prime}+i}$ uses

$$
\operatorname{rotate}\left(u, \psi_{\left\lfloor\frac{t_{B}}{2 N^{\prime}}\right\rfloor \bmod \operatorname{lcm}\left(2 N^{\prime}, w\right) / w, i-1}^{2 t^{\prime}, w}\right)
$$

as its $\mathrm{CH}$ sequence, where $t_{B}$ is the BS's local clock time (i.e. according to the BS's local clock, it is the $t_{B^{-}}$ th timeslot).

- $\forall 1 \leq j \leq U, s_{j}=u$.

An example illustrating the $\mathrm{CH}$ sequences of A-Broadcast when $N^{\prime}=4$ is shown in Figure 4.

Theorem 9 evaluates the performance of A-Broadcast. As shown in Theorem 9,
- A-Broadcast has zero broadcast latency.

- It achieves full delivery channel diversity. And the interval (i.e. $2 N^{\prime}$ ) is bounded-within every $2 N^{\prime}$ timeslots, it achieves full delivery channel diversity.

- The delivery ratio is $\frac{1}{N^{\prime}}$.

- In every timeslot, every user can receive broadcast delivery from at least $2 q \mathrm{BS}$ radios, and on average $\frac{R}{N^{\prime}}$ radios.

Theorem 9. $\mathcal{M}=(\mathcal{B}, \mathcal{U})$ is an $A M B$ system that implements A-Broadcast, where $|\mathcal{B}| \geq 2 N^{\prime}$. Then

1) The broadcast latency is 0.

2) Within every $2 N^{\prime}$ slots, it achieves full delivery channel diversity.

3) The delivery ratio $\rho=\frac{1}{N^{\prime}}$.

4) In every timeslot, $\forall s \in \mathcal{U}, s$ can have successful deliveries with at least $2 q$ radios in $\mathcal{B}$.

5) In every timeslot, $\forall s \in \mathcal{U}, s$ can have successful deliveries with $\frac{R}{N^{\prime}}=2 q+\frac{w}{N^{\prime}}$ radios in $\mathcal{B}$ on average.

2) L-Broadcast when $R<2 N^{\prime}$ : If the number of BS broadcast radios $R$ is less than $2 N^{\prime}$, we can also use the balance sequence to achieve delivery channel diversity and maximize delivery ratio. We design a ELP-based protocol, LBroadcast, as follows:

- $\forall 1 \leq i \leq R$, from timeslot $\left\lfloor\frac{t_{B}}{2 N^{\prime}}\right\rfloor$ to timeslot $\left\lfloor\frac{t_{B}}{2 N^{\prime}}\right\rfloor+$ $\left(2 N^{\prime}-1\right)$, radio $r_{i}$ uses

$$
\operatorname{rotate}\left(u, \psi_{\left\lfloor\frac{t_{B}}{2 N^{\prime}}\right\rfloor \bmod \operatorname{lcm}\left(2 N^{\prime}, R\right) / R, i-1}^{2 t^{\prime}}\right)
$$

as its $\mathrm{CH}$ sequence, where $t_{B}$ is the BS's local clock time (i.e. according to the BS's local clock, it is the $t_{B^{-}}$ th timeslot).

- $\forall 1 \leq j \leq U, s_{j}=u$.

An example illustrating the $\mathrm{CH}$ sequences of L-Broadcast when $N^{\prime}=4$ is shown in Figure 5 . 


\begin{tabular}{|c|c|c|c|c|c|c|c|c|c|c|c|c|c|c|c|c|c|c|c|c|c|c|c|c|c|c|c|c|c|c|c|c|c|}
\hline \multirow{2}{*}{$\begin{array}{l}\text { Frame index } \\
\qquad \operatorname{Radio} \mathbf{r}_{1}\end{array}$} & \multicolumn{8}{|c|}{ 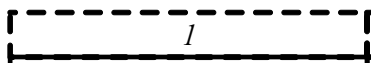 } & \multicolumn{8}{|c|}{5} & \multicolumn{8}{|c|}{5} & \multicolumn{8}{|c|}{$1----------$} & \\
\hline & $\mathbf{0}$ & $\mathbf{0}$ & 3 & 1 & 2 & 1 & 3 & 2 & $\mathbf{0}$ & $\mathbf{0}$ & 3 & 1 & 2 & 1 & 3 & 2 & $\mathbf{0}$ & $\mathbf{0}$ & 3 & 1 & 2 & 1 & 3 & 2 & $\mathbf{0}$ & $\mathbf{0}$ & 3 & 1 & 2 & 1 & 3 & 2 & \\
\hline Radio $\mathbf{r}_{2}$ & $\mathbf{0}$ & 3 & 1 & 2 & 1 & 3 & 2 & 0 & $\mathbf{0}$ & 3 & 1 & 2 & 1 & 3 & 2 & 0 & $\mathbf{0}$ & 3 & 1 & 2 & 1 & 3 & 2 & 0 & $\mathbf{0}$ & 3 & 1 & 2 & 1 & 3 & 2 & 0 & \\
\hline Radio $\mathbf{r}_{3}$ & 3 & 1 & 2 & 1 & 3 & 2 & 0 & 0 & 3 & 1 & 2 & 1 & 3 & 2 & 0 & 0 & 3 & 1 & 2 & 1 & 3 & 2 & 0 & 0 & 3 & 1 & 2 & 1 & 3 & 2 & 0 & 0 & \\
\hline $\operatorname{Radio} \mathbf{r}_{4}$ & 1 & 2 & 1 & 3 & 2 & $\mathbf{0}$ & 0 & 3 & 1 & 2 & 1 & 3 & 2 & $\mathbf{0}$ & 0 & 3 & 1 & 2 & 1 & 3 & 2 & $\mathbf{0}$ & 0 & 3 & 1 & 2 & 1 & 3 & 2 & $\mathbf{0}$ & 0 & 3 & \\
\hline Radio $\mathbf{r}_{5}$ & 2 & 1 & 3 & 2 & 0 & $\mathbf{0}$ & 3 & 1 & 2 & 1 & 3 & 2 & 0 & $\mathbf{0}$ & 3 & 1 & 2 & 1 & 3 & 2 & O & $\mathbf{0}$ & 3 & 1 & 2 & 1 & 3 & 2 & 0 & $\mathbf{0}$ & 3 & 1 & \\
\hline Radio $\mathbf{r}_{6}$ & 1 & 3 & 2 & 0 & 0 & 3 & 1 & 2 & 1 & 3 & 2 & 0 & 0 & 3 & 1 & 2 & 1 & 3 & $\mid 2$ & 0 & 0 & 3 & 1 & 2 & 1 & 3 & 2 & 0 & 0 & 3 & 1 & 2 & \\
\hline $\operatorname{Radio} \mathbf{r}_{7}$ & 3 & 2 & $\mathbf{0}$ & 0 & 3 & 1 & 2 & 1 & 3 & 2 & $\mathbf{0}$ & 0 & 3 & 1 & 2 & 1 & 3 & 2 & $\mathbf{0}$ & 0 & 3 & 1 & 2 & 1 & 3 & 2 & $\mathbf{0}$ & 0 & 3 & 1 & 2 & 1 & \\
\hline $\operatorname{Radio} \mathbf{r}_{8}$ & 2 & $\mathbf{0}$ & $\mathbf{0}$ & 3 & 1 & 2 & 1 & 3 & 2 & $\mathbf{0}$ & $\mathbf{0}$ & 3 & 1 & 2 & 1 & 3 & 2 & $\mathbf{0}$ & $\mathbf{0}$ & 3 & 1 & 2 & 1 & 3 & 2 & $\mathbf{0}$ & $\mathbf{0}$ & 3 & 1 & 2 & 1 & 3 & \\
\hline Radio $\mathbf{r}_{9}$ & $\mathbf{0}$ & $\mathbf{0}$ & 3 & 1 & 2 & 1 & 3 & 2 & 3 & 1 & 2 & 1 & 3 & 2 & 0 & 0 & 2 & 1 & 3 & 2 & O & $\mathbf{0}$ & 3 & 1 & 3 & 2 & $\mathbf{0}$ & 0 & 3 & 1 & 2 & 1 & \\
\hline Radio $\mathbf{r}_{10}$ & $\mathbf{0}$ & 3 & 1 & 2 & 1 & 3 & 2 & 0 & 1 & 2 & 1 & 3 & 2 & $\mathbf{0}$ & 0 & 3 & 1 & 3 & 2 & 0 & 0 & 3 & 1 & 2 & 2 & $\mathbf{0}$ & $\mathbf{0}$ & 3 & 1 & 2 & 1 & 3 & \\
\hline Radio $s_{1}$ & 3 & 1 & 2 & 1 & 3 & 2 & 0 & 0 & 3 & 1 & 2 & 1 & 3 & 2 & 0 & 0 & 3 & 1 & 2 & 1 & 3 & 2 & 0 & 0 & 3 & 1 & 2 & 1 & 3 & 2 & 0 & 0 & \\
\hline Radio $s_{2}$ & 1 & 3 & 2 & 0 & 0 & 3 & 1 & 2 & 1 & 3 & 2 & 0 & 0 & 3 & 1 & 2 & 1 & 3 & 2 & 0 & 0 & 3 & 1 & 2 & 1 & 3 & 2 & 0 & 0 & 3 & 1 & 2 & \\
\hline
\end{tabular}

Figure 4: Example illustrating the $\mathrm{CH}$ sequences of A-Broadcast when $N^{\prime}=4$. Radios $r_{1}, r_{2}, r_{3}, \ldots, r_{10}$ are the BS's broadcast radios and there are two users- radio $s_{1}$ and radio $s_{2}$. The clock of radio $s_{1}$ is 2 timeslots behind (or 6 timeslots ahead of) that of the BS while the clock of radio $s_{2}$ is 3 timeslots ahead of (or 5 timeslots behind) that of the BS. The blocks with red/blue fill pattern represents the timeslots in which radio $s_{1} / s_{2}$ receives successful broadcast delivery from the BS, respectively, while the blocks with gray fill pattern represents the timeslots in which both users (radios $s_{1}$ and $s_{2}$ ) receive successful broadcast delivery from the BS simultaneously. We can observe that both users can receive broadcast delivery from at least $2 q=2\left\lfloor\frac{10}{8}\right\rfloor=2$ and on average $\frac{R}{N^{\prime}}=\frac{10}{4}=2.5$ BS's broadcast radios every timeslot and achieve full broadcast channel diversity.

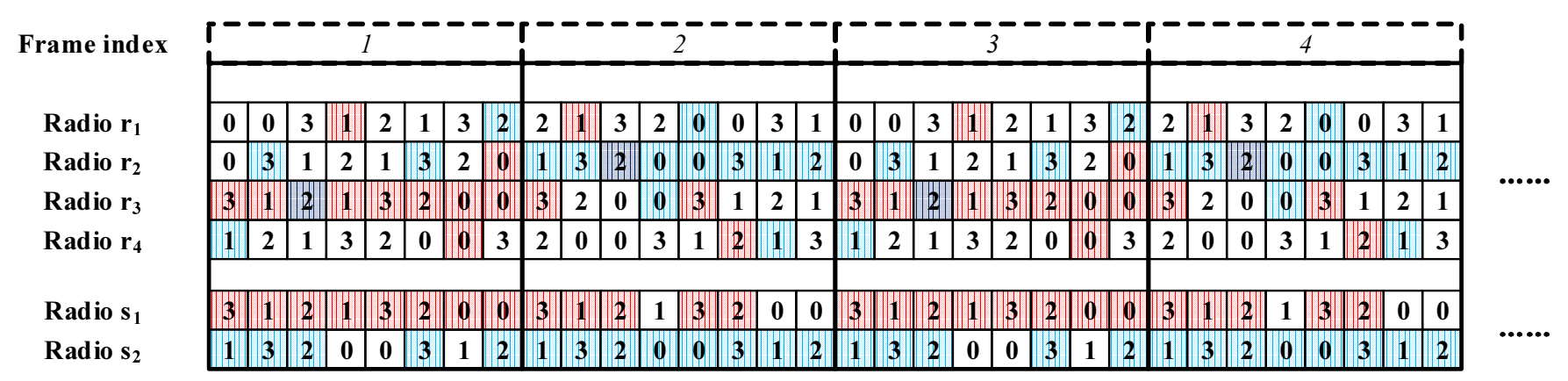

Figure 5: Example illustrating the $\mathrm{CH}$ sequences of L-Broadcast when $N^{\prime}=4$. Radios $r_{1}, r_{2}, r_{3}, r_{4}$ are the BS's broadcast radios and there are two users - radio $s_{1}$ and radio $s_{2}$. The clock of radio $s_{1}$ is 2 timeslots behind (or 6 timeslots ahead of) that of the BS while the clock of radio $s_{2}$ is 3 timeslots ahead of (or 5 timeslots behind) that of the BS. The blocks with red/blue fill pattern represents the timeslots in which radio $s_{1} / s_{2}$ receives successful broadcast delivery from the BS, respectively, while the blocks with gray fill pattern represents the timeslots in which both users (radios $s_{1}$ and $s_{2}$ ) receive successful broadcast delivery from the BS simultaneously. We can observe that both users can receive broadcast delivery from $\frac{R}{N^{\prime}}=\frac{4}{4}=1 \mathrm{BS}$ 's broadcast radio on average and achieve full delivery channel diversity every $2 N^{\prime} \cdot\left\lceil\frac{2 N^{\prime}}{R}\right\rceil=8 \cdot \frac{8}{4}=16$ timeslots.

Theorem 10 analyzes the performance of an AMB system that implements L-Broadcast. Note that S-Broadcast mentioned above is a special case of L-Broadcast. As shown in Theorem 10,

- The broadcast latency of the AMB system that implements L-Broadcast is at most $2 N^{\prime}-1$.

- It achieves full delivery channel diversity. And the interval (i.e. $2 N^{\prime} \cdot\left\lceil\frac{2 N^{\prime}}{R}\right\rceil$ ) is bounded-it achieves full delivery channel diversity every $2 N^{\prime} \cdot\left\lceil\frac{2 N^{\prime}}{R}\right\rceil$ slots.

- In every timeslot, every user can receive broadcast delivery from $\frac{R}{N^{\prime}}$ radios on average.
Theorem 10. $\mathcal{M}=(\mathcal{B}, \mathcal{U})$ is an $A M B$ system that implements L-Broadcast, where $|\mathcal{B}|<2 N^{\prime}$. Then

1) $\forall s \in \mathcal{U}$, the broadcast latency is at most $2 N^{\prime}-1$.

2) It achieves full delivery channel diversity every $2 N^{\prime}$. $\left\lceil\frac{2 N^{\prime}}{R}\right\rceil$ slots.

3) The delivery ratio $\rho=\frac{1}{N^{\prime}}$.

4) In every timeslot, $\forall s \in \mathcal{U}$, s can have broadcast delivery with $\frac{R}{N^{\prime}}$ radios in $\mathcal{B}$ on average. 


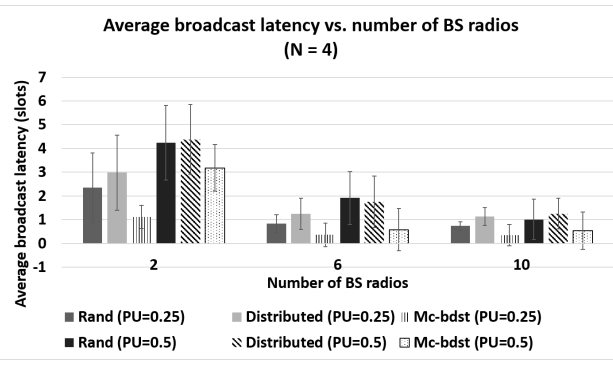

Figure 6: The average broadcast latency versus the number of broadcast radios at the base station $(N=4)$, with a $95 \%$ confidence interval attached to each bar.

\section{PERFormance EVAluation}

\section{A. Simulation Settings}

In this section, we compare the performance of the proposed Mc-Broadcast protocol and other existing protocols, including the distributed broadcast protocol (or simply called "distributed") proposed in [19] and the random channel hopping scheme, via simulation results. In each simulated network cell, the BS has $R$ broadcast radios available; a number of $U$ users are connected to the BS, and each user has a single radio interface; each radio can access $N$ broadcast channels (i.e., the number of broadcast channels available to the network is $N$ ). The $\mathrm{BS}$ or its connected users generate their $\mathrm{CH}$ sequences using the agreed broadcast scheme (i.e., either McBroadcast, the distributed protocol or the random channel hopping protocol) and perform $\mathrm{CH}$ in accordance with the sequences. Once two nodes hop onto the same channel that is free of primary user signals, the broadcast delivery between them is successful.

Traffic model. We simulated a number of $X$ primary transmitters operating on $X$ channels independently, and these channels were randomly chosen in each simulation run. In most existing work, it is assumed that a primary user transmitter follows a "busy/idle" transmission pattern on a licensed channel [6], [8], and we assume the same traffic pattern here. That is, the busy period has a fixed length of $b$ timeslots, and the idle period follows an exponential distribution with a mean of $l$ timeslots. A channel is considered "unavailable" when PU signals are present in it. The intensity of primary user traffic can be characterized as $P U=\frac{X}{N} \cdot \frac{b}{l+b}$.

Random clock drift. In a CR network, the BS and the user may lose clock synchronization or even link connectivity at any time when they experience the broadcast failure problem due to primary user affection. Hence, the clock of the BS and those of the users are not necessarily synchronized. In each simulation run, each secondary node (the BS and the users) determines its clock time independently of other nodes. Note that the radios of the BS are synchronized, and there is a random clock drift between the BS and any of its connected users.

\section{B. Impact of Simulation Parameters}

To show the impact of the number of broadcast radios $R$, we conduct a set of simulations by varying $R$ ( $R$ can be

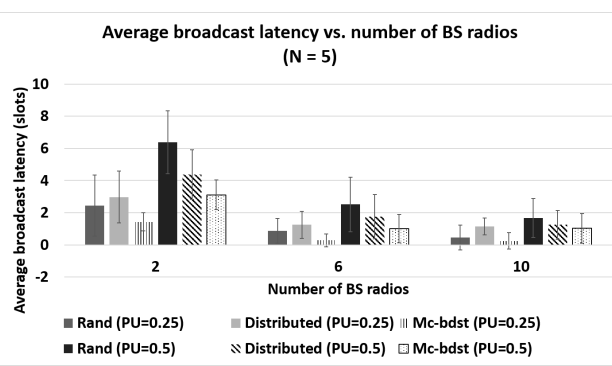

Figure 7: The average broadcast latency versus the number of broadcast radios at the base station $(N=5)$, with a $95 \%$ confidence interval attached to each bar.

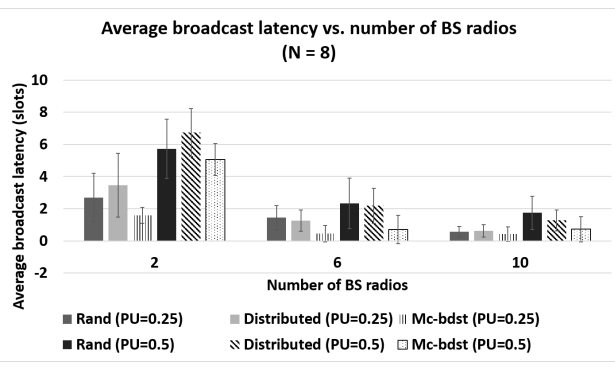

Figure 8: The average broadcast latency versus the number of broadcast radios at the base station $(N=8)$, with a $95 \%$ confidence interval attached to each bar.

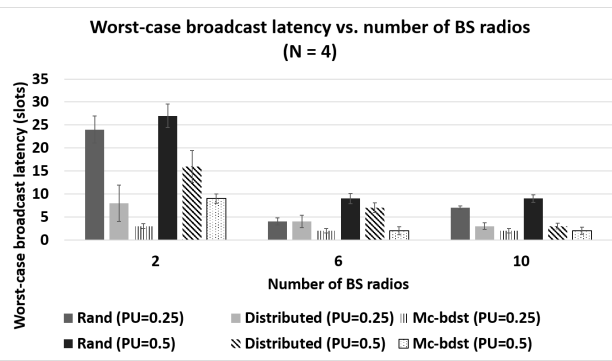

Figure 9: The worst-case broadcast latency versus the number of broadcast radios at the base station $(N=4)$, with a $95 \%$ confidence interval attached to each bar.

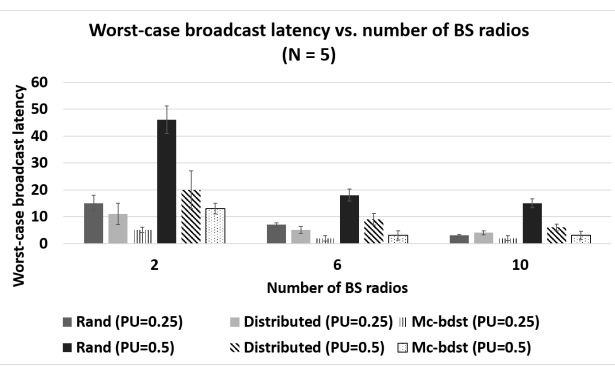

Figure 10: The worst-case broadcast latency versus the number of broadcast radios at the base station $(N=5)$, with a $95 \%$ confidence interval attached to each bar. 


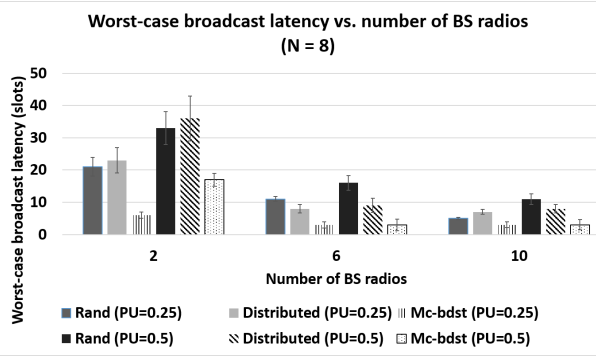

Figure 11: The worst-case broadcast latency versus the number of broadcast radios at the base station $(N=8)$, with a $95 \%$ confidence interval attached to each bar.

2, 6 and 8) under different scenarios in which the number of channels available to the network $N$ can be 4,5 , and 8 . In our simulations, each network has $U=1000$ user nodes. PU traffic $P U$ ranges from 0 (no PU activity) to 0.5 and its activity pattern is described in Sec. VI-A. Specifically, in the traffic model prescribed in Sec. VI-A, $X$ is a randomly chosen integer in $[1, N]$ and $l$ is randomly chosen from $[1,2 N]$, then according to the desired PU traffic $P U$, we determine $b$ such as $b=\frac{l}{1-P U \cdot N / X}-l$. Our proposed Mc-Broadcast protocol and other existing channel hopping protocols are compared. We show the impact of the number of broadcast radios in terms of the average broadcast latency (Fig. 6, 7 and 8) and the maximum broadcast latency (i.e. the worst-case performance, Fig. 9, 10 and 11). Additionally, we add 95\% confidence intervals to these figures.

1) Impact of the Number of Broadcast Radios: Average broadcast latency. Fig. 6,7 and 8 show the simulation results with respect to the average broadcast latency under the conditions $N=4,5$ and 8 , respectively. It is illustrated that as the number of broadcast radios increases, it takes fewer timeslots on average before the first successful delivery for both schemes under different PU traffic. This implies that a greater number of broadcast radios is conducive to mitigating the average broadcast latency.

It is noteworthy that for different numbers of available channels and different PU traffic, the average latency of McBroadcast is smaller than those of other existing broadcast protocols.

Maximum broadcast latency. Fig. 9,10 and 11 show the simulation results with respect to the maximum (worst-case) broadcast latency, which characterizes the worst-case performance of broadcast schemes, under the conditions $N=4,5$ and 8 , respectively. These results support the observation that as the number of broadcast radios increases, it takes fewer timeslots in the worst case before the first successful delivery under different primary user traffic patterns. Similarly, it is noteworthy that the proposed Mc-Broadcast scheme outperforms other existing protocols in the worst case regardless of different numbers of available channels and primary user traffic patterns.

Limit for the number of broadcast radios. There is a limit for the number of broadcast numbers. There exists an optimal number of radios in terms of cost and latency reduction performance. The theoretical optimal number of radios is twice the channel number. Although generally a greater number of broadcast radios is conducive to mitigating broadcast latency, according to the simulation results, there is only marginal improvement in terms of cost and latency reduction performance if the $\mathrm{BS}$ is equipped with more than the theoretical optimal number of radios. For example, if a network has 4 broadcast channels and the BS has been equipped with 8 radios, its performance almost remains the same when adding more radios to the BS.

2) Impact of PU Traffic Patterns: From Fig. 6, 7, 8, 9, 10 and 11, we can observe that the increase of PU traffic intensity causes a degradation in performance (in terms of both the average broadcast latency and the maximum broadcast latency) of both schemes. However, under the same PU traffic pattern and with the numbers of available channels and broadcast radios fixed, the proposed Mc-Broadcast scheme outperforms other existing broadcast protocols both on average and in the worst case.

\section{Delivery Ratio}

In this set of simulations, we measure the average delivery ratios (Table I) and the minimum (worst-case) delivery ratios (Table II) if a network uses the proposed Mc-Broadcast scheme, the random channel hopping scheme or the distributed broadcast protocol proposed in [19] under different PU traffic patterns. We simulate five networks (numbered from Network 1 to Network 5) in which the BS is equipped with 8 broadcast radios and there are $N=8$ broadcast channels with PU traffic ranging from 0 to 0.5 . In each simulated network, there are $U=1000$ user nodes. The setting of traffic model is described in Sec. VI-A. Specifically, in the traffic model prescribed in Sec. VI-A, $X$ is a randomly chosen integer in $[1, N]$ and $l$ is randomly selected from $[1,2 N]$, then according to the desired PU traffic $P U$, we determine $b$ such as $b=\frac{l}{1-P U \cdot N / X}-l$.

The delivery ratio is the percentage of the number of slots in which successful broadcast delivery occurs in a period. The average delivery ratio of a network is the average of the delivery ratios between the BS and each user node in this network. The minimum (worst-case) delivery ratio of a network is the minimum of the delivery ratios between the BS and each user node.

We can observe from Table I that with the same PU traffic, the average delivery ratios of the three simulated broadcast protocols come very close. Despite that, Mc-broadcast has the following advantages:

- Mc-Broadcast significantly outperforms the other two existing protocols in the worst-case performance (i.e. the minimum delivery ratio). Table II shows that Mcbroadcast has an advantage of $20 \%$ to $40 \%$ over the random channel hopping protocol.

- Mc-Broadcast's performance is very stable. Table I and Table II show that its worst-case performance coincides with its average performance regardless of different PU activity. Furthermore, the worst-case/average delivery ratio that Mc-broadcast maintains with PU traffic being $P U \in[0,1]$ is exactly the theoretical optimal value $\frac{1-P U}{N^{\prime}}$ proposed in Theorem 9 and Theorem 10. In contrast, the 
other existing protocols have obvious variation as to their worst-case performance.

\section{CONCLUSIONS}

In this paper, we propose an efficient broadcast protocol that allows a network base station to broadcast over multiple channels via a channel hopping process such that the broadcasts can be successfully delivered to secondary users. The channel hopping sequence is generated using a mathematical construct called Langford pairing. Our approach is novel in that

- It significantly reduces the broadcast latency and achieves full broadcast channel diversity between the BS and the users while their clocks are asynchronous.

- It guarantees the upper-bounded interval to achieve full broadcast channel diversity.

- Its performance is very stable in view of the worst-case performance that coincides with the average performance.

Our analytical and simulation results show that the proposed method reduces the broadcast latency and achieves full broadcast channel diversity and it is robust to the broadcast failure (broadcast link breakage) caused by primary user activities under various network conditions.

\section{ACKNOWLEDGEMENTS}

This work was partially supported by NSFC under grant number 61201245, Specialized Research Fund for the Doctoral Program of Higher Education (SRFDP) under grant 20120001120128, Beijing Natural Science Foundation (4143062), and SKLSDE-2014KF of State Key Laboratory of Software Development Environment.

\section{APPENDIX}

\section{Proof of Theorem 3}

Proof: If $k \equiv l\left(\bmod 2 N^{\prime}\right)$, then it is obvious that rotate $(u, k)=\operatorname{rotate}(u, l)$,

$$
\mathcal{C}(\operatorname{rotate}(u, k), \operatorname{rotate}(u, l))=\left\{0,1,2, \ldots, N^{\prime}-1\right\},
$$

and that $\mathcal{T}($ rotate $(u, k)$, rotate $(u, l))=\left\{0,1,2,3, \ldots, 2 N^{\prime}-\right.$ $1\}$.

Now we consider the case where $k-l \equiv g \not \equiv 0\left(\bmod 2 N^{\prime}\right)$, $|g| \leq N^{\prime}$. We have $\mathcal{C}($ rotate $(u, k)$, rotate $(u, l))=$ $\mathcal{C}($ rotate $(u, k-l), u)=\mathcal{C}($ rotate $(u, g), u)$. And $\forall 0 \leq j \leq$ $T-1, \operatorname{rotate}(u, g)_{j}=u_{j+g \bmod T}$, where $\operatorname{rotate}(u, g)_{j}$ denotes the $j$-th timeslot of the $\mathrm{CH}$ sequence rotate $(u, g)$.

If $g>0$, we have $1 \leq g \leq N^{\prime}$ and $0 \leq g-1 \leq N^{\prime}-1$. By the definition of ELP, there exists $i_{0}, j_{0} \in\left[0,2 N^{\prime}-1\right]$ such that $i_{0}<j_{0}$ and $u_{i_{0}}=u_{j_{0}}=g-1$. Thus we have $j_{0}-i_{0}=g$ and

$$
\text { rotate }(u, g)_{i_{0}}=u_{i_{0}+g}=u_{j_{0}}=g-1 .
$$

Since rotate $(u, g)_{i_{0}}=g-1$ and we have proved that $u_{i_{0}}=$ $g-1$, thus we obtain that

$$
g-1 \in \mathcal{C}(\text { rotate }(u, g), u)=\mathcal{C}(\text { rotate }(u, k), \operatorname{rotate}(u, l))
$$

Suppose $x \in \mathcal{C}(\operatorname{rotate}(u, k)$, rotate $(u, l))=$ $\mathcal{C}($ rotate $(u, g), u)$, then $\exists 0 \leq i_{0} \leq 2 N^{\prime}-1$ s.t. rotate $(u, g)_{i_{0}}=u_{i_{0}+g}=u_{i_{0}}=x$, and by the definition of ELP, $x+1=\left(i_{0}+g\right)-i_{0}=g, x=g-1$. Therefore $\mathcal{C}($ rotate $(u, k)$, rotate $(u, l))=\{|g|-1\}$.

If $g<0$, we have $1 \leq-g \leq N^{\prime}, 0 \leq-g \leq N^{\prime}-1$, and $\mathcal{C}($ rotate $(u, g), u)=\mathcal{C}(u$, rotate $(u,-g))$. And we reduce it to the case where $g>0$. Hence $\{|g|-1\}=\{(-g)-$ $1\}=\mathcal{C}(u, \operatorname{rotate}(u,-g))=\mathcal{C}($ rotate $(u, g)$, rotate $(u))=$ $\mathcal{C}($ rotate $(u, k)$, rotate $(u, l))$.

\section{Proof of Corollary 4}

Proof: By Theorem 3, if $k-l \equiv 0\left(\bmod 2 N^{\prime}\right)$,

$$
\mid \mathcal{C}(\text { rotate }(u, k), \text { rotate }(u, l)) \mid \geq N^{\prime} \geq 1 ;
$$

and if $k-l \not \equiv 0\left(\bmod 2 N^{\prime}\right)$,

$$
\mid \mathcal{C}(\text { rotate }(u, k), \text { rotate }(u, l)) \mid=1 \text {. }
$$

Thus we conclude that $\forall k, l \in \mathbb{Z}$,

$$
\mid \mathcal{C}(\text { rotate }(u, k), \text { rotate }(u, l)) \mid \geq 1 \text {. }
$$

\section{Proof of Theorem 5}

Proof: If $k \equiv l\left(\bmod 2 N^{\prime}\right)$ (e.g. Frame 3 in Figure $\left.3 \mathrm{a}\right)$, then it is obvious that $\operatorname{rotate}(u, k)=\operatorname{rotate}(u, l)$ and that

$$
\mathcal{T}(\text { rotate }(u, k), \text { rotate }(u, l))=\left\{0,1,2,3, \ldots, 2 N^{\prime}-1\right\} .
$$

Now we consider the case where $k-l \equiv g \not \equiv 0\left(\bmod 2 N^{\prime}\right)$ , $|g| \leq N^{\prime}$.

By Theorem 3, $\mid \mathcal{T}(\operatorname{rotate}(u, k)$, rotate $(u, l) \mid \geq 1$ and $\mathcal{C}($ rotate $(u, k)$, rotate $(u, l))=\{|g|-1\}$, i.e., the delivery channel $h=|g|-1$ and $\exists 0 \leq i_{0} \leq 2 N^{\prime}-1$ s.t. rotate $(u, k)_{i_{0}}=\operatorname{rotate}(u, l)_{i_{0}}=h$. There are only two $h$ 's in $\operatorname{rotate}(u, k)$ and $\operatorname{rotate}(u, l)$, respectively. Without loss of generality, suppose that the other $h$ in rotate $(u, k)$ is rotate $(u, k)_{\left[i_{0}-(h+1)\right] \bmod 2 N^{\prime}}$, and the other $h$ in $\operatorname{rotate}(u, l)$ is $\operatorname{rotate}(u, l)_{\left[i_{0}+(h+1)\right] \bmod 2 N^{\prime}}$.

If $i_{0}+(h+1) \equiv i_{0}-(h+1)\left(\bmod 2 N^{\prime}\right)$, then we have $2(h+1) \equiv 2|g| \equiv 0\left(\bmod 2 N^{\prime}\right)$. Since $g \neq 0$, we obtain that $|g|=N^{\prime}$. Therefore if $|g|<N^{\prime}$, we have $i_{0}+(h+1) \not \equiv i_{0}-$ $(h+1)\left(\bmod 2 N^{\prime}\right)$ and $\mid \mathcal{T}($ rotate $(u, k)$, rotate $(u, l)) \mid=1$; if $|g|=N^{\prime}$ (e.g. Frame 3 in Figure $3 \mathrm{~b}$ ), then $i_{0}+(h+1) \equiv$ $i_{0}-(h+1)\left(\bmod 2 N^{\prime}\right)$, and we have $\operatorname{rotate}(u, k)_{i_{0}}=$ rotate $(u, l)_{i_{0}}=h$ and $\operatorname{rotate}(u, k)_{\left[i_{0}-(h+1)\right] \bmod 2 N^{\prime}}=$

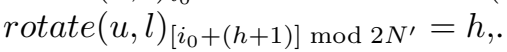

Therefore $\mid \mathcal{T}($ rotate $(u, k)$, rotate $(u, l)) \mid=2$.

\section{Proof of Theorem 6}

Proof: If $\forall k \in \mathbb{Z}, \exists 1 \leq f \leq F$, s.t. $o_{f}-k \equiv 0$ $\left(\bmod 2 N^{\prime}\right)$, then $\forall k, l \in \mathbb{Z}, \exists 1 \leq f \leq F$, s.t. $o_{f}-(l-k) \equiv 0$ $\left(\bmod 2 N^{\prime}\right)$. We obtain that

$$
\begin{aligned}
N^{\prime} & \geq \mid \mathcal{C}(\operatorname{rotate}(r, k), \operatorname{rotate}(s, l) \mid \\
& =\mid \mathcal{C}(\operatorname{rotate}(r, k-l), s \mid \\
& \geq\left|\mathcal{C}\left(\operatorname{rotate}\left(u, o_{f}-(l-k)\right), u\right)\right| \\
& =|\mathcal{C}(u, u)|=N^{\prime} .
\end{aligned}
$$

Thus $D I V(\mathcal{M})=N^{\prime}$.

If $\exists k_{0} \in \mathbb{Z}$, s.t. $\forall 1 \leq f \leq F, o_{f}-k_{0} \neq 0\left(\bmod 2 N^{\prime}\right)$, by Theorem $3, \mathcal{C}\left(\right.$ rotate $\left(u, o_{f}\right)$, rotate $\left.(u, k)\right)=\left\{\delta\left(o_{f}-k_{0}\right)\right\}$. Thus $\mid \mathcal{C}\left(r\right.$, rotate $\left.\left(s, k_{0}\right)\right)|=|\left\{\delta\left(o_{f}-k_{0}\right)\right\}_{1 \leq f \leq F} \mid \leq F$, 


\begin{tabular}{|c|c|c|c|c|c|c|c|c|c|}
\hline \multirow[b]{2}{*}{ Network } & \multicolumn{3}{|c|}{$P U=0$} & \multicolumn{3}{|c|}{$P U=0.25$} & \multicolumn{3}{|c|}{$P U=0.5$} \\
\hline & $\begin{array}{c}\text { Mc- } \\
\text { Broadcast }\end{array}$ & Rand. & Dist. & $\begin{array}{c}\text { Mc- } \\
\text { Broadcast }\end{array}$ & Rand. & Dist. & $\begin{array}{c}\text { Mc- } \\
\text { Broadcast }\end{array}$ & Rand. & Dist. \\
\hline 1 & 0.125 & 0.125756 & 0.102156 & 0.09375 & 0.093801 & 0.088193 & 0.0625 & 0.057219 & 0.059482 \\
\hline 2 & 0.125 & 0.125487 & 0.081807 & 0.09375 & 0.094214 & 0.096395 & 0.0625 & 0.064242 & 0.064952 \\
\hline 3 & 0.125 & 0.124920 & 0.121225 & 0.09375 & 0.093950 & 0.086093 & 0.0625 & 0.062298 & 0.059874 \\
\hline 4 & 0.125 & 0.124958 & 0.131584 & 0.09375 & 0.093069 & 0.091691 & 0.0625 & 0.063116 & 0.069454 \\
\hline 5 & 0.125 & 0.125042 & 0.090916 & 0.09375 & 0.094237 & 0.095495 & 0.0625 & 0.063719 & 0.054845 \\
\hline
\end{tabular}

Table I: Average delivery ratio.

\begin{tabular}{|c|c|c|c|c|c|c|c|c|c|}
\hline \multirow{3}{*}{ Network } & \multicolumn{3}{|c|}{$P U=0$} & \multicolumn{3}{c|}{$P U=0.25$} & \multicolumn{3}{c|}{$P U=0.5$} \\
\cline { 2 - 10 } & $\begin{array}{c}\text { Mc- } \\
\text { Broadcast }\end{array}$ & Rand. & Dist. & $\begin{array}{c}\text { Mc- } \\
\text { Broadcast }\end{array}$ & Rand. & Dist. & $\begin{array}{c}\text { Mc- } \\
\text { Broadcast }\end{array}$ & Rand. & Dist. \\
\hline 1 & 0.125 & 0.092773 & 0.119845 & 0.09375 & 0.067383 & 0.0767121 & 0.0625 & 0.036133 & 0.0609353 \\
\hline 2 & 0.125 & 0.099609 & 0.107964 & 0.09375 & 0.068359 & 0.0695130 & 0.0625 & 0.045573 & 0.0482546 \\
\hline 3 & 0.125 & 0.105469 & 0.116589 & 0.09375 & 0.07666 & 0.0862363 & 0.0625 & 0.04541 & 0.0536983 \\
\hline 4 & 0.125 & 0.109375 & 0.112300 & 0.09375 & 0.072266 & 0.0799273 & 0.0625 & 0.044531 & 0.0437375 \\
\hline 5 & 0.125 & 0.103841 & 0.098455 & 0.09375 & 0.077474 & 0.0886291 & 0.0625 & 0.048828 & 0.0569861 \\
\hline
\end{tabular}

Table II: Minimum delivery ratio.

and $\min _{k \in \mathbb{Z}}\left|\left\{\delta\left(o_{f}-k\right)\right\}_{1 \leq f \leq F}\right| \leq D I V(\mathcal{M}) \leq \mid\left\{\delta\left(o_{f}-\right.\right.$ $\left.\left.k_{0}\right)_{1 \leq f \leq F}\right\} \mid \leq F$.

\section{Proof of Theorem 7}

Proof: We group every $2 N^{\prime}$ slots of $r$ into a frame, i.e., $\left\{r_{i}\right\}_{0 \leq i<2 N^{\prime}}$ forms the first frame, $\left\{r_{i}\right\}_{2 N^{\prime} \leq i<4 N^{\prime}}$ forms the second frame, etc., as illustrated in Figures $3 \mathrm{a}$ and $3 \mathrm{~b}$. The segment of $r$ in the $f$ th frame, denoted by $r[f]$, is rotate $(u, f-1)$, and the segment of $s$ in the $f$ th frame, denoted by $s[f]$, is always $\operatorname{rotate}\left(u, k_{0}\right)$ for some $k_{0} \in \mathbb{Z}$ for all frames. For example, in Figure $3 \mathrm{~b}, s[f]=\operatorname{rotate}(u, 2)$ for $\forall f \in \mathbb{N}$. By Corollary 4 , in the first frame, broadcast delivery can occur between $r[1]=u$ and $s[1]=\operatorname{rotate}\left(u, k_{0}\right)$. Thus the broadcast latency is at most the frame size $2 N^{\prime}-1$. Since every period is homogeneous, with loss of generality, we consider the first period, i.e. from the first frame to the $2 N^{\prime}$ th frame. For $1 \leq f \leq 2 N^{\prime},\left\{\left[(f-1)-k_{0}\right] \bmod 2 N^{\prime}\right\}$ is a permutation of $\left\{0,1,2,3, \ldots, 2 N^{\prime}-1\right\}$, by Theorem 5 ,

$$
\begin{aligned}
& |\mathcal{T}(r, s)| \\
= & \sum_{f=1}^{n}|\mathcal{T}(r[f], s[f])| \\
= & \sum_{f=1}^{n}\left|\mathcal{T}\left(\operatorname{rotate}(u, f-1), \operatorname{rotate}\left(u, k_{0}\right)\right)\right| \\
= & \sum_{g(f)=0} \mid \mathcal{T}\left(\operatorname{rotate}(u, f-1), \operatorname{rotate}\left(u, k_{0}\right) \mid\right. \\
& +\sum_{g(f)=N^{\prime}} \mid \mathcal{T}\left(\operatorname{rotate}(u, f-1), \operatorname{rotate}\left(u, k_{0}\right) \mid\right. \\
& +\sum_{0<g(f)<N^{\prime}} \mid \mathcal{T}\left(\operatorname{rotate}(u, f-1), \operatorname{rotate}\left(u, k_{0}\right) \mid\right. \\
= & 1 \cdot 2 N^{\prime}+1 \cdot 2+\left(2 N^{\prime}-2\right) \cdot 1=4 N^{\prime},
\end{aligned}
$$

where $g(f) \equiv(f-1)-k_{0}\left(\bmod 2 N^{\prime}\right)$ and $|g(f)| \leq N^{\prime}$. Thus $\rho(r, s)=\frac{|\mathcal{T}(r, s)|}{T}=\frac{4 N^{\prime}}{2 N^{\prime} \cdot 2 N^{\prime}}=\frac{1}{N^{\prime}}$. Since $\exists 1 \leq f_{0} \leq F$ s.t. $\left(f_{0}-1\right)-k_{0} \equiv 0\left(\bmod 2 N^{\prime}\right)\left(\right.$ in Figure $\left.3 \mathrm{a}, f_{0}=3\right)$, we have rotate $\left(u, f_{0}-1\right)=\operatorname{rotate}\left(u, k_{0}\right)$ and $N^{\prime} \geq|\mathcal{C}(r, s)| \geq$ $\mid \mathcal{C}\left(\right.$ rotate $\left(u, f_{0}-1\right)$, rotate $\left.\left(u, k_{0}\right)\right) \mid=N^{\prime}$. Thus $|\mathcal{C}(r, s)|=$ $N^{\prime}$.

\section{Proof of Theorem 9}

Proof: Given an arbitrary $s \in \mathcal{U}$, we call the first $2 N^{\prime}$ timeslots the first frame. The segment of $s$ in the first frame is $\operatorname{rotate}\left(u, k_{0}\right)$ for some $k_{0} \in \mathbb{Z}$. The segments of radios $r_{1}, r_{2}, r_{3}, \ldots, r_{2 N^{\prime}}$ in the first frame are rotate $\left(u, l_{0}\right)$, rotate $\left(u, l_{0}+1\right)$, rotate $\left(u, l_{0}+\right.$ $2), \ldots, \operatorname{rotate}\left(u, l_{0}+2 N^{\prime}-1\right) . \exists r_{0} \in \mathcal{B}$ s.t. the segment of $r_{0}$ in the first frame is exactly rotate $\left(u, k_{0}\right)$. Thus $\mathcal{B}$ and $s$ can have successful broadcast delivery in the first frame and therefore the broadcast latency is 0 .

For arbitrarily given $2 N^{\prime}$ consecutive slots, within which the segments of $\mathrm{CH}$ sequences $r_{1}, r_{2}, r_{3}, \ldots, r_{2 N^{\prime}}$ are $\operatorname{rotate}\left(u, m_{0}\right), \operatorname{rotate}\left(u, m_{0}+1\right), \operatorname{rotate}\left(u, m_{0}+\right.$ $2), \ldots$,rotate $\left(u, m_{0}+2 N^{\prime}-1\right)$ for some $m_{0} \in \mathbb{Z}$, respectively, and the segment of $s$ is rotate $\left(u, n_{0}\right)$ for some $n_{0} \in \mathbb{Z}$, and we have $\exists r_{0} \in \mathcal{B}$ s.t. the segment of $r_{0}$ is exactly rotate $\left(u, n_{0}\right)$. Thus the full delivery diversity is achieved.

The period $T$ of $\mathcal{M}$ is $\frac{2 N^{\prime} \operatorname{lcm}\left(2 N^{\prime}, w\right)}{w}$. Within a period, without loss of generality, suppose for $i \in\left[1,2 q N^{\prime}\right], r_{i}=$ rotate $\left(u,(i-1) \bmod 2 N^{\prime}\right)$, for $i \in\left[2 q N^{\prime}+1, R\right]$, we have $r_{i}=\operatorname{rotate}\left(u, \psi_{\left.\frac{t_{B}}{2 N^{\prime}}\right\rfloor \bmod \operatorname{lcm}(n, k) / k, i-s q N^{\prime}-1}^{2 N^{\prime},}\right)$, and $s=\operatorname{rotate}(u, \Delta)$ due to the clock drift. By Theorem 5 ,

$$
\begin{aligned}
& \sum_{i=1}^{2 q N^{\prime}}\left|\mathcal{T}\left(r_{i}, s\right)\right| \\
= & q \sum_{i=1}^{2 N^{\prime}}\left|\mathcal{T}\left(r_{i}, s\right)\right| \\
= & \frac{T}{2 N^{\prime}} \cdot q \sum_{i=1}^{2 N^{\prime}}|\mathcal{T}(\operatorname{rotate}(u, i-1), \operatorname{rotate}(u, \Delta))| \\
= & \frac{T}{2 N^{\prime}} \cdot q\left[1 \cdot 2 N^{\prime}+1 \cdot 2+\left(2 N^{\prime}-2\right) \cdot 1\right] \\
= & \frac{T}{2 N^{\prime}} \cdot q\left(4 N^{\prime}\right)=2 q T,
\end{aligned}
$$

where $g(i) \equiv(i-1)-\Delta \bmod 2 N^{\prime}$ and $|g(i)| \leq N^{\prime}$. By 
Theorem 5 and Lemma 8,

$$
\begin{aligned}
& \sum_{i=1}^{w}\left|\mathcal{T}\left(r_{2 q N^{\prime}+i}, s\right)\right| \\
= & \frac{\operatorname{lcm}\left(2 N^{\prime}, w\right)}{2 N^{\prime}} \cdot \sum_{i=1}^{2 N^{\prime}}|\mathcal{T}(\operatorname{rotate}(u, i), \operatorname{rotate}(u, \Delta))| \\
= & \frac{\operatorname{lcm}\left(2 N^{\prime}, w\right)}{2 N^{\prime}} \cdot\left[1 \cdot 2 N^{\prime}+1 \cdot 2+\left(2 N^{\prime}-2\right) \cdot 1\right] \\
= & \frac{\operatorname{lcm}\left(2 N^{\prime}, w\right)}{2 N^{\prime}} \cdot 4 N^{\prime} \\
= & 2 \cdot \frac{w T}{2 N^{\prime}} \\
= & \frac{w T}{N^{\prime}}
\end{aligned}
$$

where $g(i) \equiv(i-1)-\Delta \bmod 2 N^{\prime}$ and $|g(i)| \leq N^{\prime}$. Thus the delivery ratio

$$
\begin{aligned}
\rho & =\frac{\sum_{i=1}^{R}\left|\mathcal{T}\left(r_{i}, s\right)\right|}{R T} \\
& =\frac{\sum_{i=1}^{2 q N^{\prime}}\left|\mathcal{T}\left(r_{i}, s\right)\right|+\sum_{i=1}^{w}\left|\mathcal{T}\left(r_{2 q N^{\prime}+i}, s\right)\right|}{R T} \\
& =\frac{1}{R} \cdot\left(2 q+w / N^{\prime}\right) \\
& =\frac{1}{N^{\prime}} .
\end{aligned}
$$

For an arbitrarily given timeslot $t \in[0, T-1]$, suppose the $t$-th timeslot of $\mathrm{CH}$ sequence $s$ is $s_{t}=h \in\left[0, N^{\prime}-1\right]$. Suppose $t=2 q^{\prime} N^{\prime}+r^{\prime}$, where $0 \leq r^{\prime}<2 N^{\prime}$, and suppose the segments of radios in $r_{1}, r_{2}, r_{3}, \ldots, r_{2 N^{\prime}}$ from timeslot $2 q^{\prime} N^{\prime}$ to timeslot $2 q^{\prime} N^{\prime}+\left(2 N^{\prime}-1\right)$ are rotate $\left(u, m_{0}\right)$, rotate $\left(u, m_{0}+\right.$ $1)$, rotate $\left(u, m_{0}+2\right), \ldots$, rotate $\left(u, m_{0}+2 N^{\prime}-1\right)$, respectively. By the definition of ELP, there exists $0 \leq i<j<2 N^{\prime}$ such that rotate $\left(u, m_{0}\right)_{i}=\operatorname{rotate}\left(u, m_{0}\right)_{j}=h$. Thus for $k \in\left[0,2 N^{\prime}-1\right]$, we have

$$
\text { rotate }\left(u, m_{0}+k\right)_{(i-k) \bmod 2 N^{\prime}}=\operatorname{rotate}\left(u, m_{0}\right)_{i}=h
$$

and

$$
\text { rotate }\left(u, m_{0}+k\right)_{(j-k) \bmod 2 N^{\prime}}=\operatorname{rotate}\left(u, m_{0}\right)_{j}=h .
$$

Let $k_{1} \triangleq\left(i-r^{\prime}\right) \bmod 2 N^{\prime}, k_{2} \triangleq\left(j-r^{\prime}\right) \bmod 2 N^{\prime}$, and we have $\operatorname{rotate}\left(u, m_{0}+k_{1}\right)_{r^{\prime}}=\operatorname{rotate}\left(u, m_{0}+i-\right.$ $\left.r^{\prime}\right)_{r^{\prime}}=\operatorname{rotate}\left(u, m_{0}\right)_{i-r^{\prime}+r^{\prime}}=\operatorname{rotate}\left(u, m_{0}\right)_{i}=h$, and similarly rotate $\left(u, m_{0}+k_{2}\right)_{r^{\prime}}=\operatorname{rotate}\left(u, m_{0}+j-r^{\prime}\right)_{r^{\prime}}=$ rotate $\left(u, m_{0}\right)_{j-r^{\prime}+r^{\prime}}=\operatorname{rotate}\left(u, m_{0}\right)_{j}=h$. Since $i \neq j$, $k_{1} \neq k_{2}$. We obtain that in the timeslot $t, s$ can have broadcast delivery with radio $r_{k_{1}+1}$ and radio $r_{k_{2}+1}$. Similarly, in the timeslot $t, s$ can broadcast delivery with these $2 q$ radios $r_{k_{1}+1+2 p N^{\prime}}, r_{k_{2}+1+2 p N^{\prime}}$, where $p \in[0, q-1]$. In an average timeslot, $s$ can have broadcast delivery with

$$
\frac{\sum_{i=1}^{R}\left|\mathcal{T}\left(r_{i}, s\right)\right|}{T}=R \rho=\frac{R}{N^{\prime}}=2 q+\frac{w}{N^{\prime}}
$$

radios in $\mathcal{B}$.

\section{Proof of Theorem 10}

Proof: The period $T$ of $\mathcal{M}$ is $\frac{2 N^{\prime} \cdot \operatorname{lcm}\left(2 N^{\prime}, R\right)}{R}$.

For an arbitrarily given $s \in \mathcal{U}$, we group every $2 N^{\prime}$ slots of the BS into a frame, i.e., the first $2 N^{\prime}$ slots according to the
BS's clock form the first frame, the next $2 N^{\prime}$ slots form the second frame, etc., and thus the segment of $r_{1}$ in the first frame is $u$ and the segment of $s$ in the first frame is $\operatorname{rotate}(u, \Delta)$ for some $\Delta \in \mathbb{Z}$. By Corollary $4, r_{1}$ and $s$ can have broadcast delivery within the first frame, and thus the broadcast latency is at most $2 N^{\prime}-1$.

Since every period is homogeneous, without loss of generality, we consider the first period, i.e. from the first frame to the $F$ th frame, where $F=\frac{T}{2 N^{\prime}}=\frac{\operatorname{lcm}\left(2 N^{\prime}, R\right)}{R}$.

Without loss generality, suppose

$r_{i}[f]=\operatorname{rotate}\left(u, \psi_{f-1, i-1}^{2 N^{\prime}, R}\right)=[(f-1) R+(i-1)] \bmod 2 N^{\prime}$ and $s[f]=\operatorname{rotate}(u, \Delta)$ for some $\Delta \in \mathbb{Z}$ due to the clock drift. Thus if $f$ is incremented from $f_{0}$ to $f_{0}+\left(\left\lceil\frac{2 N^{\prime}}{R}\right\rceil-1\right)$ and $i$ is incremented from 0 to $R-1, \psi_{f-1, i-1}^{2 N^{\prime}, R}$ can take values of $0,1,2,3, \ldots, 2 N^{\prime}-1$. There exists $\tilde{f} \in\left[f_{0}, f_{0}+\left(\left\lceil\frac{2 N^{\prime}}{R}\right\rceil-1\right)\right]$ and $\tilde{i} \in[0, R-1]$ such that $r_{\tilde{i}}[\tilde{f}]=\operatorname{rotate}(u, \Delta)=s[f]$. Therefore it achives full diversity every $\left\lceil\frac{2 N^{\prime}}{R}\right\rceil$ frames, i.e., $2 N^{\prime} \cdot\left\lceil\frac{2 N^{\prime}}{R}\right\rceil$ slots.

By Theorem 5 and Lemma 8,

$$
\begin{aligned}
& \sum_{i=1}^{R}\left|\mathcal{T}\left(r_{i}, s\right)\right| \\
= & \frac{\operatorname{lcm}\left(2 N^{\prime}, R\right)}{2 N^{\prime}} \cdot \sum_{i=1}^{2 N^{\prime}} \mid \mathcal{T}(\text { rotate }(u, i), \operatorname{rotate}(u, \Delta)) \mid \\
= & \frac{\operatorname{lcm}\left(2 N^{\prime}, R\right)}{2 N^{\prime}} \cdot\left[1 \cdot 2 N^{\prime}+1 \cdot 2+\left(2 N^{\prime}-2\right) \cdot 1\right] \\
= & \frac{\operatorname{lcm}\left(2 N^{\prime}, R\right)}{2 N^{\prime}} \cdot 4 N^{\prime} \\
= & 2 \operatorname{lcm}\left(2 N^{\prime}, R\right) \\
= & \frac{R T}{N^{\prime}}
\end{aligned}
$$

where $g(i) \equiv(i-1)-\Delta \bmod 2 N^{\prime}$ and $|g(i)| \leq N^{\prime}$. Therefore the delivery density

$$
\rho=\frac{\sum_{i=1}^{R}\left|\mathcal{T}\left(r_{i}, s\right)\right|}{R T}=\frac{R T}{R T \cdot N^{\prime}}=\frac{1}{N^{\prime}} .
$$

In an average timeslots, $s$ can have broadcast delivery with

$$
\frac{\sum_{i=1}^{R}\left|\mathcal{T}\left(r_{i}, s\right)\right|}{T}=R \rho=\frac{R}{N^{\prime}}
$$

radios in $\mathcal{B}$.

\section{REFERENCES}

[1] C. J. L. Arachchige, S. Venkatesan, R. Chandrasekaran, and N. Mittal, "Minimal time broadcasting in cognitive radio networks," in Proc. ICDCN, pp. 364-375, 2011.

[2] T. Baykas, M. Kasslin, M. Cummings, H. Kang, J. Kwak, R. Paine, A. Reznik, R. Saeed and S. J. Shellhammer, "Developing a standard for TV white space coexistence: technical challenges and solution approaches," IEEE Wireless Communications, vol. 19, no. 1, pp. 10-22, 2012.

[3] L. Berlemann, C. Hoymann, G. Hiertz, and B. Walke. Minimum-Latency Broadcast Scheduling for Cognitive Radio Networks Proc. of IEEE Secon 2013.

[4] C. Cormio and K. R. Chowdhury, "Common control channel design for cognitive radio wireless ad hoc networks using adaptive frequency hopping," Ad Hoc Networks, vol. 8, no. 4, pp. 430-438, 2010.

[5] K. Etemad and L. Wang, "Multicast and broadcast multimedia services in mobile WiMAX networks," IEEE Communications Magazine, vol. 47, no. 10, pp. 84-91, 2009. 
[6] S. Geirhofer, L. Tong, and B. Sadler. "Cognitive medium access: constraining interference based on experimental models," IEEE J. Selected Areas of Comm., vol. 26, no. 1, pp. 95-105, Jan. 2008.

[7] M. Gruber and D. Zeller, "Multimedia broadcast multicast service: new transmission schemes and related challenges," IEEE Communications Magazine, vol. 49, no. 12, pp. 176-181, 2011.

[8] S. Huang, X. Liu, and Z. Ding, "Optimal transmission strategies for dynamic spectrum access in cognitive radio networks," IEEE Transactions on Mobile Computing, vol. 8, no. 12, pp. 1636-1648, Dec. 2009.

[9] R. Jain, The Art of Computer Systems Performance Analysis. WileyInterscience, Apr. 1991.

[10] T. Jiang, W. Xiang, H. H. Chen and Q. Ni, "Multicast broadcast services support in OFDMA-Based WiMAX systems', IEEE Communications Magazine, vol. 45, no. 8, pp. 78-86, 2007.

[11] Y. R. Kondareddy and P. Agrawal, "Selective broadcasting in multi-hop cognitive radio networks," IEEE Sarnoff Symposium, pp. 1-5, 2008.

[12] M. Kornfeld, "DVB-H-the emerging standard for mobile data communication," IEEE International Symposium on Consumer Electronics, pp. 193-198, 2004.

[13] C. D. Langford, Problem. The Mathematical Gazette, vol. 42, no. 341, p. 228 , Oct. 1958

[14] L. Lazos, S. Liu, and M. Krunz, "Spectrum opportunity-based control channel assignment in cognitive radio networks," in Proc. IEEE SECON, pp. 1-9, 2009

[15] Z. Lin, H. Liu, X. Chu, and Y-W. Leung, "Jump-stay based channelhopping algorithm with guaranteed rendezvous for cognitive radio networks," in Proc. IEEE INFOCOM, pp. 2444-2452, 2011.

[16] J. E. Simpson, "Langford sequences: perfect and hooked," Discrete Mathematics, vol. 44, no.1, pp. 97-104, 1983.

[17] Y. Song, J. L. Xie and X. Wang, "A Novel Unified Analytical Model for Broadcast Protocols in Multi-hop Cognitive Radio Ad Hoc Networks," IEEE Transactions on Mobile Computing, vol. 99, no. 1, p. 1, 2013.

[18] Y. Song and J. Xie, "A QoS-based broadcast protocol for multi-hop cognitive radio ad hoc networks under blind information," IEEE Global Telecommunications Conference, pp. 1-5, 2011.

[19] Y. Song and J. Xie, "A distributed broadcast protocol in multi-hop cognitive radio ad hoc networks without a common control channel," Proc. IEEE INFOCOM, pp. 2273-2281, 2012.

[20] W. Whitt, "The Efficiency of One Long Run versus Independent Replications in Steady-State Simulation," Management Science, vol. 37, no. 6, pp. 645-666, 1991.

[21] D. Yang, J. Shin, and C. Kim, "Deterministic rendezvous scheme in multichannel access networks," Electronics Letters, vol. 46, no. 20, pp. $1402-1404,2010$

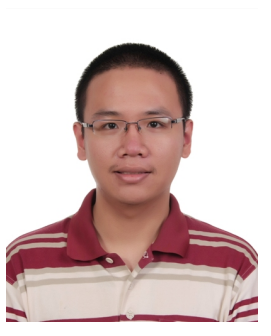

Lin Chen is currently a Ph.D. student in the Department of Electrical Engineering, Graduate School of Arts and Sciences, Yale University. He received his B.S. degree in computer science from Peking University in 2014. His research interests focus on wireless networks and network theory.

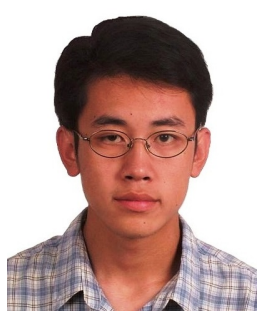

Kaigui Bian received his Ph.D. degree in computer engineering from Virginia Tech, Blacksburg, USA in 2011. He is currently an assistant professor in the Institute of Network Computing and Information Systems, School of EECS at Peking University. His research interests include mobile computing, cognitive radio networks, network security and privacy.

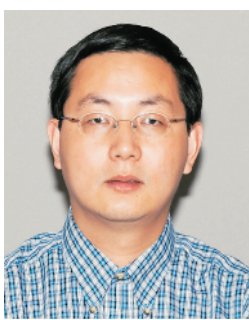

Dr. Xiaojiang (James) Du is currently an associate professor in the Department of Computer and Information Sciences at Temple University. Dr. Du received his B.S. and M.S. degree in electrical engineering from Tsinghua University, Beijing, China in 1996 and 1998, respectively. He received his M.S. and Ph.D. degree in electrical engineering from the University of Maryland College Park in 2002 and 2003, respectively. Dr. Du was an Assistant Professor in the Department of Computer Science at North Dakota State University between August 2004 and July 2009, where he received the Excellence in Research Award in May 2009. His research interests are security, cloud computing, wireless networks, computer networks and systems. He has published over 130 journal and conference papers in these areas. Dr. Du has been awarded more than $\$ 5 \mathrm{M}$ research grants from the US National Science Foundation (NSF), Army Research Office, Air Force Research Lab, NASA, the Commonwealth of Pennsylvania, and Amazon. He serves on the editorial boards of four international journals. Dr. Du will serve as the Lead Chair of the Communication and Information Security Symposium of the IEEE ICC 2015, and a Co-Chair of the Mobile and Wireless Networks Track of the IEEE WCNC 2015. He was the Chair of the Computer and Network Security Symposium of the IEEE/ACM International Wireless Communication and Mobile Computing conference 2006 - 2010. He is (was) a Technical Program Committee (TPC) member of several premier ACM/IEEE conferences such as INFOCOM (2007-2015), IM, NOMS, ICC, GLOBECOM, WCNC, BroadNet, and IPCCC. Dr. Du is a Senior Member of IEEE and a Life Member of ACM.

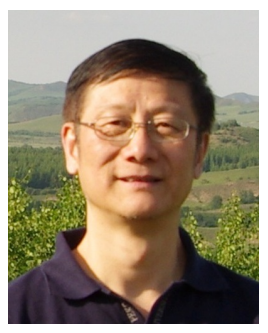

Xiaoming $\mathbf{L i}$ received his Ph.D. in computer science from Stevens Institute of Technology (USA) in 1986 and now is a Professor at Peking University. His research interests include Web search and mining, online social network analysis. He is an editor of Concurrency and Computation, and an editor of Networking Science. 